\begin{tabular}{|c|l|}
\hline Title & Global classification of curves on the symplectic plane \\
\hline Author(s) & Ishikawa, Goo \\
\hline Citation & Hokkaido University Preprint Series in Mathematics, 824, 1-24 \\
\hline Issue Date & 2007 \\
\hline DOI & 10.14943/83974 \\
\hline Doc URL & http://hdl.handle.net/2115/69632 \\
\hline Type & bulletin (article) \\
\hline File Information & pre824.pdf \\
\hline
\end{tabular}

Instructions for use 


\title{
Global classification of curves on the symplectic plane
}

\author{
Goo ISHIKAWA*
}

\section{Introduction.}

In [17], we considered the local classification of plane curves on the symplectic plane. In particular, we introduced the number "symplectic defect", which represents the difference of two natural equivalence relations on plane curves, the equivalence by diffeomorphisms and that by symplectomorphisms. For an immersion, two equivalence relations coincide, so the symplectic defect is null. For complicated singularities, the symplectic defects turn out to be positive.

In this paper we consider the global symplectic classification problem. First we give the exact classification result under symplectomorphisms, for the case of generic plane curves, namely immersions with transverse self-intersections. Then, for a given diffeomorphism class of a generic plane curve, the set of symplectic classes form the symplectic moduli space which we completely describe by its global topological term (Theorem 1.1). In the general plane curves with singularities, the difference between symplectomorphism and diffeomorphism classifications is clearly described by local symplectic moduli spaces of singularities and a global topological term. Thus, up to the classification by diffeomorphisms, the global problem is reduced to the local classification problem (Theorem 1.4). We introduce the symplectic moduli space of a global plane curve and the local symplectic moduli space of a plane curve singularity as quotients of mapping spaces, and we endow them with differentiable structures in a natural way. Actually we treat labelled plane curves and labelled symplectic moduli spaces. For a plane curve, we label all compact domains surrounded by it and all singular points, and consider the classification problem of plane curves isotopic to the given plane curve by symplectomorphisms preserving the labelling.

Let $f: S^{1} \rightarrow \mathbb{R}^{2}$ be a generic immersion of the circle $S^{1}$ in the symplectic plane $\mathbb{R}^{2}$ with the standard symplectic (area) form $\omega_{0}=d x \wedge d y$. Clearly the areas of domains surrounded by the curve $f\left(S^{1}\right)$ are invariant under symplectomorphisms. Thus, denoting the first Betti number of $f\left(S^{1}\right)$ by $r$, we see the

Key words: symplectomorphism, moduli space, mapping space quotient, differentiable structure 2000 Mathematics Subject Classification: Primary 58K40; Secondly 58C27, 58D15, 53Dxx.

*Partially supported by Grants-in-Aid for Scientific Research, No. 14340020. 
curves isotopic to $f$ have $r$-dimensional symplectic moduli.

We denote by $C^{\infty}\left(S^{1}, \mathbb{R}^{2}\right)$ the space of $C^{\infty}$ mappings from $S^{1}$ to $\mathbb{R}^{2}$, which has the natural action (from "right") of the group $\operatorname{Diff}^{+}\left(S^{1}\right)$ consisting of orientation-preserving diffeomorphisms on $S^{1}$. Thus $C^{\infty}\left(S^{1}, \mathbb{R}^{2}\right) / \operatorname{Diff}^{+}\left(S^{1}\right)$ denotes the space of oriented curves. The space $C^{\infty}\left(S^{1}, \mathbb{R}^{2}\right) / \operatorname{Diff}^{+}\left(S^{1}\right)$ has the action (from "left") of the group $\operatorname{Diff}^{+}\left(\mathbb{R}^{2}\right)$ (resp. $\operatorname{Symp}\left(\mathbb{R}^{2}\right)$ ) consisting of orientation-preserving diffeomorphisms (resp. symplectomorphisms) on $\mathbb{R}^{2}$. For each oriented curve $f \in C^{\infty}\left(S^{1}, \mathbb{R}^{2}\right) / \operatorname{Diff}^{+}\left(S^{1}\right)$, we denote by $\operatorname{Diff}^{+}\left(\mathbb{R}^{2}\right) f$ the orbit through $f$ via the action of Diff $\left(\mathbb{R}^{2}\right)$. Thus $\operatorname{Diff}^{+}\left(\mathbb{R}^{2}\right) f$ consists of oriented curves of form $\rho \circ f$ for orientation preserving diffeomorphisms $\rho$. Similarly the space $\operatorname{Diff}{ }^{+}\left(\mathbb{R}^{2}\right) f / \operatorname{Symp}\left(\mathbb{R}^{2}\right)$ means the quotient space by the $\operatorname{Symp}\left(\mathbb{R}^{2}\right)$-action of Diff $\left(\mathbb{R}^{2}\right) f$ in $C^{\infty}\left(S^{1}, \mathbb{R}^{2}\right) / \operatorname{Diff}^{+}\left(S^{1}\right)$. (The expression $\operatorname{Symp}\left(\mathbb{R}^{2}\right) \backslash \operatorname{Diff}{ }^{+}\left(\mathbb{R}^{2}\right) f$ would be better, but I do not adopt it.)

We call the quotient space $\operatorname{Diff}\left(\mathbb{R}^{2}\right) f / \operatorname{Symp}\left(\mathbb{R}^{2}\right)$ the symplectic moduli space of $f$ and denote it by $\mathcal{M}_{\text {symp }}(f)$. It describes the symplectic classification of a fixed isotopy class of an oriented plane curve.

To study the moduli space minutely, we label the $r$-domains surrounded by the curve $f\left(S^{1}\right)$ as $D_{1}, D_{2}, \ldots, D_{r}$ for a $f \in C^{\infty}\left(S^{1}, \mathbb{R}^{2}\right) / \operatorname{Diff}^{+}\left(S^{1}\right)$. Then, for each $\rho \in \operatorname{Diff}^{+}\left(\mathbb{R}^{2}\right)$, we label bounded $r$-domains surrounded by $(\rho \circ f)\left(S^{1}\right)$ as $\rho\left(D_{1}\right), \rho\left(D_{2}\right), \ldots, \rho\left(D_{r}\right)$ induced by the labelling for $f$. We set

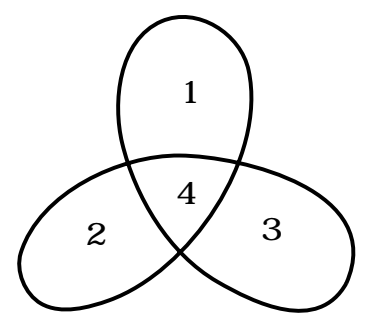

Figure 1: A labelling of a generic plane curve.

$$
\widetilde{\mathcal{M}}_{\text {symp }}(f)=\operatorname{Diff}^{+}\left(\mathbb{R}^{2}\right) / \sim_{f},
$$

where we call $\rho, \rho^{\prime} \in \operatorname{Diff}^{+}\left(\mathbb{R}^{2}\right)$ are equivalent via $f$, and write $\rho \sim_{f} \rho^{\prime}$, if the exists a symplectomorphism $\tau$ such that $\tau \circ \rho \circ f=\rho^{\prime} \circ f$ up to $\operatorname{Diff}^{+}\left(S^{1}\right)$ and $\tau$ preserves the given labelling: $\tau\left(\rho\left(D_{j}\right)\right)=\rho^{\prime}\left(D_{j}\right), 1 \leq j \leq r$. We call $\widetilde{\mathcal{M}}_{\text {symp }}(f)$ the labelled symplectic moduli space of $f$. Note that $\widetilde{\mathcal{M}}_{\text {symp }}(f)$ does not depend on the chosen labelling of $f$.

The natural projection $\pi: \widetilde{\mathcal{M}}_{\text {symp }}(f) \rightarrow \mathcal{M}_{\text {symp }}(f)$ is defined by $\pi([\rho])=$ $[\rho \circ f]$ and $\pi$ is a finite (at most $r !, r$ factorial) to one.

Then in this paper we show in fact: 
Theorem 1.1 If $f \in C^{\infty}\left(S^{1}, \mathbb{R}^{2}\right) / \operatorname{Diff}^{+}\left(S^{1}\right)$ is a generic immersion, then the labelled symplectic moduli space $\widehat{\mathcal{M}}_{\text {symp }}(f)$ is diffeomorphic to the relative cohomology space $H^{2}\left(\mathbb{R}^{2}, f\left(S^{1}\right), \mathbb{R}\right) \cong \mathbb{R}^{r}$.

The labelled symplectic moduli space $\widetilde{\mathcal{M}}_{\text {symp }}(f)$ has a canonical differentiable structure. We claim in Theorem 1.1 that the labelled symplectic moduli space of $f$ with the differentiable structure is diffeomorphic to $\mathbb{R}^{r}$, $r=\operatorname{dim}_{\mathbb{R}} H^{2}\left(\mathbb{R}^{2}, f\left(S^{1}\right), \mathbb{R}\right)=\operatorname{dim}_{\mathbb{R}} H_{1}\left(f\left(S^{1}\right), \mathbb{R}\right)$.

Actually we are going to give a diffeomorphism between $\widetilde{\mathcal{M}}_{\text {symp }}(f)$ and the positive cone in $H^{2}\left(\mathbb{R}^{2}, f\left(S^{1}\right), \mathbb{R}\right)$. Note that the relative cohomology group $H^{2}\left(\mathbb{R}^{2}, f\left(S^{1}\right), \mathbb{R}\right)$ over $\mathbb{R}$ is isomorphic to the vector space $H_{2}\left(\mathbb{R}^{2}, f\left(S^{1}\right), \mathbb{R}\right)^{*}=$ $\operatorname{Hom}_{\mathbb{R}}\left(H_{2}\left(\mathbb{R}^{2}, f\left(S^{1}\right), \mathbb{R}\right), \mathbb{R}\right)$. The orientation of $\mathbb{R}^{2}$ and labelling of the bounded domains surrounded by $f\left(S^{1}\right)$ give the canonical basis $\left[D_{1}\right],\left[D_{2}\right], \ldots,\left[D_{r}\right]$ of $H_{2}\left(\mathbb{R}^{2}, f\left(S^{1}\right), \mathbb{R}\right)$. The positive cone $H^{2}\left(\mathbb{R}^{2}, f\left(S^{1}\right), \mathbb{R}\right)_{>0}$ is defined by

$$
H^{2}\left(\mathbb{R}^{2}, f\left(S^{1}\right), \mathbb{R}\right)_{>0}=\left\{\alpha \in H^{2}\left(\mathbb{R}^{2}, f\left(S^{1}\right), \mathbb{R}\right) \mid \alpha\left(\left[D_{j}\right]\right)>0,1 \leq j \leq r\right\} .
$$

The diffeomorphism of $\widetilde{\mathcal{M}}_{\text {symp }}(f)$ and $H^{2}\left(\mathbb{R}^{2}, f\left(S^{1}\right), \mathbb{R}\right)_{>0}$ is given actually by the mapping

$$
\varphi: \widetilde{\mathcal{M}}_{\text {symp }}(f) \rightarrow H^{2}\left(\mathbb{R}^{2}, f\left(S^{1}\right), \mathbb{R}\right)_{>0},
$$

defined by

$$
\varphi:[\rho] \mapsto\left(\left[D_{j}\right] \mapsto \int_{\rho\left(D_{j}\right)} \omega_{0}=\int_{D_{j}} \rho^{*} \omega_{0}\right) .
$$

$1 \leq j \leq r, \omega_{0}=d x \wedge d y$.

The symplectic moduli space $\mathcal{M}_{\text {symp }}(f)=\operatorname{Diff}\left(\mathbb{R}^{2}\right) f / \operatorname{Symp}\left(\mathbb{R}^{2}\right)$ is obtained as a quotient of $\widetilde{\mathcal{M}}_{\text {symp }}(f)$. A symmetry of a generic immersion $f$ : $S^{1} \rightarrow \mathbb{R}^{2}$ is an orientation preserving diffeomorphism $\rho: \mathbb{R}^{2} \rightarrow \mathbb{R}^{2}$ such that $\rho \circ f=f \circ \sigma$ for some $\sigma \in \operatorname{Diff}^{+}\left(S^{1}\right)$. We denote by $S_{f}$ the group of symmetries of $f$. Then $S_{f}$ induces a subgroup $G_{f}$ of the permutation group $S_{r}$ of the $r$ bounded domains of $\mathbb{R}^{2} \backslash f\left(S^{1}\right)$. Then $G_{f}$ naturally acts on $H_{2}\left(\mathbb{R}^{2}, f\left(S^{1}\right), \mathbb{R}\right)$ and on $H_{2}\left(\mathbb{R}^{2}, f\left(S^{1}\right), \mathbb{R}\right)^{*} \cong H^{2}\left(\mathbb{R}^{2}, f\left(S^{1}\right), \mathbb{R}\right) \cong \mathbb{R}^{r}$ as permutation of coordinates.

Example 1.2 Let $f$ be a generic immersion as in Figure 1: Then $G_{f} \subset S_{4}$ is a cyclic group of order 3 .

By Theorem 1.1, we have the following:

Corollary 1.3 The symplectic moduli space $\mathcal{M}_{\mathrm{symp}}(f)$ is diffeomorphic to the finite quotient $\mathbb{R}^{r} / G_{f}$ of $\mathbb{R}^{r}$.

In fact, the action of $G_{f} \subset S_{r}$ commutes with the diffeomorphism $\left(\mathbb{R}_{>0}\right)^{r} \rightarrow$ $\mathbb{R}^{r}$ defined by $\left(x_{1}, \ldots, x_{r}\right) \mapsto\left(\log x_{1}, \ldots, \log x_{r}\right)$. 
We denote by $\mathcal{M}_{\text {symp }}$ the whole orbit space of $C^{\infty}\left(S^{1}, \mathbb{R}^{2}\right)$ by the $\operatorname{Diff}{ }^{+}\left(S^{1}\right) \times$ $\operatorname{Symp}\left(\mathbb{R}^{2}\right)$-action (the right-left-symplectic action):

$$
\mathcal{M}_{\text {symp }}:=C^{\infty}\left(S^{1}, \mathbb{R}^{2}\right) /\left(\operatorname{Diff}^{+}\left(S^{1}\right) \times \operatorname{Symp}\left(\mathbb{R}^{2}\right)\right) .
$$

Note that $\mathcal{M}_{\text {symp }}$ is a non-Hausdorff space, with respect to the quotient topology of $C^{\infty}$ topology. The non-Hausdorffness comes from the adjacencies of $\operatorname{Diff}^{+}\left(\mathbb{R}^{2}\right) \times \operatorname{Diff}^{+}\left(S^{1}\right)$-orbits.

Besides the singular topology, we have the decomposition

$$
\mathcal{M}_{\text {symp }}=\bigcup \mathcal{M}_{\text {symp }}(f),
$$

where $f$ runs over representatives of the set of isotopy types of oriented plane curves. We ask the structure of each stratum $\mathcal{M}_{\text {symp }}(f)$ itself. Then Corollary 1.3 guarantees that each "open stratum" $\mathcal{M}_{\text {symp }}(f)$ is a finite quotient of an affine space where $f$ is a generic immersion.

The method to provide a "differentiable structure" to a mapping space quotient (a moduli space) should be not unique [7][26]. For instance, consider the problem how to define a differentiable structure on a mapping space $C^{\infty}(N, M)$ itself for $C^{\infty}$ manifolds $N$ and $M$. Then one of the standard methods seems to define, first, Fréchet differentiable functions on the Banach manifolds $C^{r}(N, M)$, for each finite $r$, and regard $C^{\infty}(N, M)$ as the inverse limit of $C^{r}(N, M)$ to define the structure sheaf of differentiable functions on it. However in this paper we apply another method: We regard Fréchet differential of a functional as a kind of "total differential". Then we could consider, instead, "partial differentials". Namely, to define differentiable functions on $C^{\infty}(N, M)$, first we define the notion of finite dimensional differentiable families in $C^{\infty}(N, M)$ by the very classical and natural manner. Then we call a function on $C^{\infty}(N, M)$ differentiable if its restriction to any finite dimensional family in $C^{\infty}(N, M)$ is of class $C^{\infty}$ in the ordinary sense. See $\S 2$.

Theorem 1.1 is generalised to more singular curves. To state the generalisation, first we treat the local case.

A multi-germ $f=f_{y_{0}}:\left(S^{1}, S\right) \rightarrow\left(\mathbb{R}^{2}, y_{0}\right)$ at a finite set $S \subset S^{1}$ is called of finite codimension (or $\mathcal{A}$-finite) in the sense of Mather if $f_{y_{0}}$ is determined by its finite jet up to diffeomorphisms (or $\mathcal{A}$ equivalence). See [24][31]. We denote by $\operatorname{Diff}_{0}^{+}\left(S^{1}, S\right)$ the group of orientation preserving diffeomorphism-germs $\left(S^{1}, S\right)$ fixing $S$ pointwise, and we treat $f_{y_{0}}$ up to $\operatorname{Diff}_{0}^{+}\left(S^{1}, S\right)$. Then the local image of $f_{y_{0}}$ divides $\left(\mathbb{R}^{2}, 0\right)$ into several domains. We label them. Then, for any orientation-preserving diffeomorphism-germ $\rho \in \operatorname{Diff}^{+}\left(\mathbb{R}^{2}, y_{0}\right)$, the labelling of $\rho \circ f$ is induced. Two diffeomorphism-germs $\rho, \rho^{\prime} \in \operatorname{Diff}^{+}\left(\mathbb{R}^{2}, y_{0}\right)$ are equivalent via $f_{y_{0}}$, and write $\rho \sim_{f} \rho^{\prime}$, if there exists a symplectomorphism-germ $\tau \in$ $\operatorname{Symp}\left(\mathbb{R}^{2}, 0\right)$ such that $\tau \circ \rho \circ f_{y_{0}}=\rho^{\prime} \circ f_{y_{0}}$ up to $\operatorname{Diff}_{0}^{+}\left(S^{1}, S\right)$ and $\tau$ preserves the labelling. Thus we define the local labelled symplectic moduli space by

$$
\widetilde{\mathcal{M}}_{\text {symp }}\left(f_{y_{0}}\right):=\operatorname{Diff}^{+}\left(\mathbb{R}^{2}, y_{0}\right) / \sim_{f} \text {. }
$$


Moreover we define the local symplectic moduli space by

$$
\mathcal{M}_{\text {symp }}\left(f_{y_{0}}\right):=\operatorname{Diff}^{+}\left(\mathbb{R}^{2}, y_{0}\right) f_{y_{0}} / \operatorname{Symp}\left(\mathbb{R}^{2}, y_{0}\right) .
$$

Note that the space of map-germs

$$
C^{\infty}\left((N, S),\left(M, y_{0}\right)\right):=\left\{f:(N, S) \rightarrow\left(M, y_{0}\right) C^{\infty} \text { map-germs }\right\}
$$

is a quotient space of $C^{\infty}(N, M)$, so also it has the differentiable structure. In particular $\widetilde{\mathcal{M}}_{\text {symp }}\left(f_{y_{0}}\right)$ and $\mathcal{M}_{\text {symp }}\left(f_{y_{0}}\right)$ are mapping space quotient have natural differentiable structures. Moreover there exists the canonical projection $\pi: \widetilde{\mathcal{M}}_{\text {symp }}\left(f_{y_{0}}\right) \rightarrow \mathcal{M}_{\text {symp }}\left(f_{y_{0}}\right)$ defined by $\pi([\rho])=[\rho \circ f]$ modulo $\operatorname{Diff}_{0}^{+}\left(S^{1}, S\right)$.

Now returning to the global case, we consider again an oriented curve $f$ : $S^{1} \rightarrow \mathbb{R}^{2}$ up to $\operatorname{Diff}^{+}\left(S^{1}\right)$, namely, $f \in C^{\infty}\left(S^{1}, \mathbb{R}^{2}\right) / \operatorname{Diff}^{+}\left(S^{1}\right)$. Then we call $f$ of finite type if, for some (and for any) representative $f: S^{1} \rightarrow \mathbb{R}^{2}$ of $f \in C^{\infty}\left(S^{1}, \mathbb{R}^{2}\right) / \operatorname{Diff}^{+}\left(S^{1}\right)$, except for a finite number of points $y_{0} \in f\left(S^{1}\right)$, the multi-germ $f_{y_{0}}:\left(S^{1}, f^{-1}\left(y_{0}\right)\right) \rightarrow\left(\mathbb{R}^{2}, y_{0}\right)$ is a stable multi-germ, namely a single immersion-germ or a transversal two-immersion-germ, and, even if $f_{y_{0}}$ is unstable, $f^{-1}\left(y_{0}\right)$ is a finite set in $S^{1}$ and $f_{y_{0}}$ is of finite codimension. The condition means roughly that the $\operatorname{Diff}^{+}\left(\mathbb{R}^{2}\right)$-orbit through $f$ in $C^{\infty}\left(S^{1}, \mathbb{R}^{2}\right) / \operatorname{Diff}{ }^{+}\left(S^{1}\right)$ is of finite codimension.

If $f \in C^{\infty}\left(S^{1}, \mathbb{R}^{2}\right) / \operatorname{Diff}^{+}\left(S^{1}\right)$ is of finite type, then $f\left(S^{1}\right)$ divides $\mathbb{R}^{2}$ into a finite number of bounded domains and one unbounded domain. Then we define the the labelling of $f$ as the labelling of bounded domains $D_{1}, \ldots, D_{r}$ and the multiple or singular values $y_{1}, \ldots, y_{s}$ of $f$ in $\mathbb{R}^{2}$ (Figure 2, where $r=4, s=6$ ). We define, similarly to the case of generic immersions, the labelled symplectic

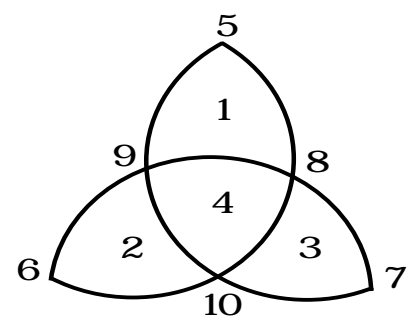

Figure 2: A labelling of a plane curve of finite type.

moduli space of plane curve $f$ of finite type by

$$
\widetilde{\mathcal{M}}_{\text {symp }}(f):=\operatorname{Diff}^{+}\left(\mathbb{R}^{2}\right) / \sim_{f},
$$

where $\rho \sim_{f} \rho^{\prime}$ if $\tau \circ \rho \circ f=\rho^{\prime} \circ f$ for some $\tau \in \operatorname{Symp}\left(\mathbb{R}^{2}\right)$ preserving the labellings induced by $\rho$ and $\rho^{\prime}$. 
Moreover we define the symplectic moduli space of plane curve $f$ of finite type by

$$
\mathcal{M}_{\text {symp }}(f):=\operatorname{Diff}^{+}\left(\mathbb{R}^{2}\right) f / \operatorname{Symp}\left(\mathbb{R}^{2}\right) .
$$

Theorem 1.4 (Localisation Theorem) If $f \in C^{\infty}\left(S^{1}, \mathbb{R}^{2}\right) / \operatorname{Diff}^{+}\left(S^{1}\right)$ is of finite type, then

$$
\widetilde{\mathcal{M}}_{\text {symp }}(f) \cong_{\text {diffeo. }} \prod_{y_{0} \in f\left(S^{1}\right)} \widetilde{\mathcal{M}}_{\text {symp }}\left(f_{y_{0}}\right) \times \mathbb{R}^{r}
$$

where $r=\operatorname{dim}_{\mathbb{R}} H^{2}\left(\mathbb{R}^{2}, f\left(S^{1}\right), \mathbb{R}\right)$. Moreover

$$
\mathcal{M}_{\text {symp }}(f) \cong_{\text {diffeo. }}\left(\prod_{y_{0} \in f\left(S^{1}\right)} \widetilde{\mathcal{M}}_{\text {symp }}\left(f_{y_{0}}\right) \times \mathbb{R}^{r}\right) / G_{f},
$$

where $G_{f} \subset S_{r^{\prime}}$ is the group induced by the symmetry group of $f, r^{\prime}$ being $r$ plus the number of unstable singular values of $f$.

Note that $\widetilde{\mathcal{M}}_{\text {symp }}\left(f_{y_{0}}\right)$ is just a point if $f_{y_{0}}$ is stable, namely if it is a single immersion-germ or a transverse self-intersection. Therefore the product in Theorem 1.4 turns out to be a finite product.

Theorem 1.4 can be regarded as the "localisation theorem" for the global labelled moduli space of the isotopy type of a singular plane curve.

The diffeomorphism between $\widetilde{\mathcal{M}}_{\text {symp }}(f)$ and the product of local symplectic moduli spaces and an open cone of $H^{2}\left(\mathbb{R}^{2}, f\left(S^{1}\right), \mathbb{R}\right)_{>0}$ is given by the mapping

$$
\Phi: \widetilde{\mathcal{M}}_{\text {symp }}(f) \rightarrow\left(\prod_{y_{0} \in f\left(S^{1}\right)} \widetilde{\mathcal{M}}_{\text {symp }}\left(f_{y_{0}}\right)\right) \times H^{2}\left(\mathbb{R}^{2}, f\left(S^{1}\right), \mathbb{R}\right)_{>0}
$$

defined by

$$
\Phi([\rho]):=\left(\left(\left[\eta_{\rho\left(y_{0}\right)} \circ \rho\right]\right)_{y_{0} \in f\left(S^{1}\right)}, \varphi([\rho])\right),
$$

where $\eta_{\rho\left(y_{0}\right)}:\left(\mathbb{R}^{2}, \rho\left(y_{0}\right)\right) \rightarrow\left(\mathbb{R}^{2}, y_{0}\right)$ is any symplectomorphism-germ. Note that $\left[\eta_{\rho\left(y_{0}\right)} \circ \rho\right] \in \widetilde{\mathcal{M}}\left(f_{y_{0}}\right)$ does not depend on the choice of $\eta_{\rho\left(y_{0}\right)}$.

Here are some examples of local (labelled) symplectic moduli spaces:

Example 1.5 (1) For the germ $f:(\mathbb{R}, 0) \rightarrow\left(\mathbb{R}^{2}, 0\right)$ of type $A_{2}$ defined by $f(t)=\left(t^{2}, t^{3}\right)$ we have $\widetilde{\mathcal{M}}_{\text {symp }}(f)=\mathcal{M}_{\text {symp }}(f)=\{\mathrm{pt}\}$.

(2) For multi-germ $f:(\mathbb{R},\{0,1\}) \rightarrow\left(\mathbb{R}^{2}, 0\right)$ (resp. $f:(\mathbb{R},\{0,1\}) \rightarrow\left(\mathbb{R}^{2}, 0\right)$; $\left.f:(\mathbb{R},\{0,1,2\}) \rightarrow\left(\mathbb{R}^{2}, 0\right)\right)$ defined by $f(t)=(t, 0)$ near $t=0$ and $f(s)=$ $(0, s-1)$ near $s=1$ (resp. $f(t)=(t, 0)$ near $t=0$ and $f(s)=\left(0,(s-1)^{2}\right)$ near $s=1 ; f(t)=(t, 0)$ near $t=0, f(s)=(0, s-1)$ near $s=1$ and $f(r)=(r-2, r-2)$ near $r=2)$ ), we have $\widetilde{\mathcal{M}}_{\text {symp }}(f)=\mathcal{M}_{\text {symp }}(f)=\{\mathrm{pt}\}$.

(3) Let $f:(\mathbb{R}, 0) \rightarrow\left(\mathbb{R}^{2}, 0\right)$ be a map-germ of type $E_{12}$ defined by $f(t)=$ $\left(t^{3}, t^{7}\right)$. Then $f$ has the symplectic normal form $\left(t^{3}, t^{7}+\lambda t^{8}\right)$. and we see $\widetilde{\mathcal{M}}_{\text {symp }}(f)=\mathcal{M}_{\text {symp }}(f)$ is diffeomorphic to $\mathbb{R}$. 
(4) Let $f:(\mathbb{R}, 0) \rightarrow\left(\mathbb{R}^{2}, 0\right)$ be a map-germ of type $W_{18}$ defined by $f(t)=$ $\left(t^{4}, t^{7}+t^{9}\right)$. Then $f$ has the symplectic normal form $\left(t^{4}, t^{7}+\lambda_{1} t^{9}+\lambda_{2} t^{13}\right), \lambda_{1}>0$, and we see $\widetilde{\mathcal{M}}_{\text {symp }}(f)=\mathcal{M}_{\text {symp }}(f)$ is diffeomorphic to $\mathbb{R}^{2}$.

(5) Let $f:(\mathbb{R}, 0) \rightarrow\left(\mathbb{R}^{2}, 0\right)$ be a map-germ of type $E_{24}$ defined by $f(t)=$ $\left(t^{3}, t^{13}+t^{14}\right)$. Then $f$ has the symplectic normal form $\left(t^{3}, t^{13}+\lambda_{1} t^{14}+\lambda_{2} t^{17}+\right.$ $\left.\lambda_{3} t^{20}\right), \lambda_{1}>0$, and we see $\widetilde{\mathcal{M}}_{\text {symp }}(f)=\mathcal{M}_{\text {symp }}(f)$ is diffeomorphic to $\mathbb{R}^{3}$.

As an application of Theorem 1.4, we have for instance:

Example 1.6 (cf. [17]). If $f: S^{1} \rightarrow \mathbb{R}^{2}$ is an injective $C^{\infty}$ map with just one singular point, where $f$ is locally diffeomorphic to $t \rightarrow\left(t^{3}, t^{13}+t^{14}\right)$ (of type $E_{24}$ ). Then the local symplectic moduli space for the singular point is diffeomorphic to $\mathbb{R}^{3}$ and the symplectic moduli space $\mathcal{M}_{\text {symp }}(f)=\operatorname{Diff}^{+}\left(\mathbb{R}^{2}\right) f / \operatorname{Symp}\left(\mathbb{R}^{2}\right)$ of $f$ is diffeomorphic to $\mathbb{R}^{3} \times \mathbb{R} \cong \mathbb{R}^{4}$.

Remark 1.7 In all known examples, we observe that the local symplectic moduli space are differentiable manifolds and the symplectic defect is interpreted as the dimension of the tangent space to the moduli space. We conjecture that this holds in general for multi-germs $f$ of finite type. Moreover, we conjecture that $\widetilde{\mathcal{M}}_{\text {symp }}(f)$ is diffeomorphic to $\mathbb{R}^{\ell}$ with $\ell=\frac{1}{2} \mu(f)-\operatorname{codim}(f)$, where $\mu(f)$ is Milnor number of $f$ and $\operatorname{codim}(f)$ is the $\mathcal{A}_{e}$-codimension of $f([31])$.

Over the complex numbers, we conjecture that similarly defined $\mathcal{M}_{\text {symp }}(f)$ is a $K(\pi, 1)$ space and the universal covering of $\mathcal{M}_{\text {symp }}(f)$ is diffeomorphic to $\mathbb{C}^{\ell}$ with $\ell=\frac{1}{2} \mu(f)-\operatorname{codim}(f)$. See $[29]$.

Similar result to Theorem 1.4 holds for curves with finite components: Let $N$ be a one dimensional closed manifold, i.e. a finite disjoint union of circles.

Theorem 1.8 If $f \in C^{\infty}\left(N, \mathbb{R}^{2}\right) / \operatorname{Diff}^{+}(N)$ is of finite type, then, for the labelled symplectic moduli space, we have

$$
\widetilde{\mathcal{M}}_{\text {symp }}(f) \cong_{\text {diffeo. }}\left(\prod_{y_{0} \in f(N)} \widetilde{\mathcal{M}}_{\text {symp }}\left(f_{y_{0}}\right)\right) \times H^{2}\left(\mathbb{R}^{2}, f(N), \mathbb{R}\right) .
$$

Moreover $\mathcal{M}_{\text {symp }}(f)$ is diffeomorphic to $\widetilde{\mathcal{M}}_{\text {symp }}(f) / G_{f}$.

Furthermore, Theorems 1.4 and 1.8 are generalised to curves with a finite number of components in a symplectic surface:

A two dimensional symplectic manifold is called a symplectic surface. A symplectic surface $(M, \omega)$ has a bounded area (resp. an unbounded area) if $\int_{M} \omega<\infty($ resp. $=\infty)$. Denote by $\operatorname{Diff}_{0}(M)\left(\operatorname{resp} . \operatorname{Symp}_{0}(M)\right)$ the group of diffeomorphisms on $M$ isotopic to the identity (resp. symplectomorphisms on $M$ isotopic to the identity through symplectomorphisms.) Then we set

$$
\widetilde{\mathcal{M}}_{\text {symp }}(f):=\operatorname{Diff}_{0}(M) / \sim_{f},
$$


where $\rho \sim_{f} \rho^{\prime}$ if there exists $\tau \in \operatorname{Symp}_{0}(f)$ preserving a labelling of $f$ and satisfying that $\tau \circ \rho \circ f=\rho^{\prime} \circ f$. Moreover we set

$$
\mathcal{M}_{\text {symp }}(f):=\operatorname{Diff}_{0}(M) f / \operatorname{Symp}_{0}(f) .
$$

Then we have

Theorem 1.9 Let $(M, \omega)$ be a connected symplectic surface, $N$ a one dimensional closed manifold and $f \in C^{\infty}(N, M) / \operatorname{Diff}^{+}(N)$ an oriented curve of finite type. Denote by $r$ the number of connected components of $M \backslash f(N)$ with bounded areas.

(1) If $(M, \omega)$ itself has an unbounded area, then

$$
\widetilde{\mathcal{M}}_{\text {symp }}(f) \cong \text { diffeo. }\left(\prod_{y_{0} \in f\left(S^{1}\right)} \widetilde{\mathcal{M}}_{\text {symp }}\left(f_{y_{0}}\right)\right) \times \mathbb{R}^{r} .
$$

(2) If $(M, \omega)$ itself has a bounded area, then

$$
\widetilde{\mathcal{M}}_{\text {symp }}(f) \cong \text { diffeo. }\left(\prod_{y_{0} \in f\left(S^{1}\right)} \widetilde{\mathcal{M}}_{\text {symp }}\left(f_{y_{0}}\right)\right) \times \mathbb{R}^{r-1} .
$$

Moreover, in any case, $\mathcal{M}_{\text {symp }}(f)$ is diffeomorphic to $\widetilde{\mathcal{M}}_{\text {symp }}(f) / G_{f}$.

Remark 1.10 (1) If $(M, \omega)$ has a bounded area, then the sum of areas of domains surrounded by the curve must be equal to the total area. This restriction reduces the dimension of the moduli space by one.

(2) If $M$ is a closed surface, then $(M, \omega)$ has a bounded area for any symplectic form $\omega$.

(3) If $(M, \omega)=\left(\mathbb{R}^{2}, \omega_{0}\right)$, the standard symplectic $\mathbb{R}^{2}$, then $r$ is equal to $\operatorname{dim}_{\mathbb{R}} H^{2}\left(\mathbb{R}^{2}, f(N), \mathbb{R}\right)$.

To describe completely the symplectic classification, we need to solve also the classification problem of plane curves by diffeomorphisms. See [1] for the global classification of generic immersions by diffeomorphisms (or homeomorphisms). For the local diffeomorphism classification (over the complex), there are several detailed studies [6][32][30][3][27][22]. Note that [3] gives the classification of simple singularities of parametric plane curves. The classification results are improved by [23][12][19] recently. Note that, in [19], we give the diffeomorphism classification of unimodal singularities of parametric plane curves, and moreover we describe concretely the symplectic moduli space of simple and unimodal plane curve singularities in the complex analytic category.

On the plane, the classification by symplectomorphisms is of course same as the classification by area-preserving diffeomorphisms. Nevertheless our classification problem seems to belong to symplectic geometry not to volume geometry, from both the motivation and the mathematical reason. In fact, the diffeomorphism invariance of symplectic defects for plane curves is naturally 
generalised to higher dimensional cases in term of Lagrangian varieties in symplectic spaces([18]). See several related results [29][21][8][5].

Naturally we can ask the similar results to Theorems of this paper, for higher dimensional cases: The symplectic classification of Lagrangian surfaces of a given Lagrangian isotopy class in $\mathbb{R}^{4}$ for instance. For that, we have to clarify on the topology (or symmetries) of generic Lagrangian immersions as well as the local classification of singularities of Lagrangian surfaces ([11][16]). Both problems seems to be very interesting.

Also, setting various geometric structures on the symplectic moduli spaces like in [13][14][2] would be very interesting problem, which is still open as far as I know.

In $\S 2$, we define a differentiable structure an any mapping space quotient.

In $\S 3$, we introduce a class of mapping space quotients which is reasonable to study for our purpose.

In $\S 4$, we define differentiable structures on the (labelled) symplectic moduli space. The symplectic classification problem of curves of fixed diffeomorphism class can be translated to the classification of forms by diffeomorphisms fixing a curve ([33][34][4][5]). In $\S 5$, we relate the space of symplectic forms with the symplectic moduli spaces. Then we give proofs of main theorems.

The starting point of the present paper is the symplectic bifurcation problem studied by the joint work [17] with S. Janeczko. I would like to thank him for his continuous encouragement. I am grateful to H. Sato and S. Izumi for their valuable comment.

\section{Differentiable structure on a mapping space quotient.}

We denote by $C^{\infty}(N, M)$ the space of $C^{\infty}$ mappings from a (finite dimensional) $C^{\infty}$ manifold $N$ to a (finite dimensional) $C^{\infty}$ manifold $M$. In this section, also $P, Q, L, K$ always designate (finite dimensional) $C^{\infty}$ manifolds respectively.

Let $X \subseteq C^{\infty}(N, M)$ be a subset. Then, such a set $X$ is a mapping space. Let $X / \sim$ be any quotient of $X$ under an equivalence relation $\sim$ on $X$. We give on the quotient space $X / \sim$ the quotient topology of $X$ with the relative topology of the $C^{\infty}$ topology on $C^{\infty}(N, M)$, not Whitney (fine) $C^{\infty}$ topology. Such space is called a mapping space quotient. Then we will endow, in the following five steps, a differentiable structure with the mapping space quotient $X$, depending just on the representation $X / \sim \leftarrow X \subseteq C^{\infty}(N, M)$. We note that the notion of differentiable structures can provided just by defining the notion of diffeomorphisms. Therefore our goal is to define the notion of "diffeomorphisms".

(i) We call a mapping $h: P \rightarrow X$ differentiable (or $C^{\infty}$ ) if there exists a $C^{\infty}$ mapping (between manifolds) $H: P \times N \rightarrow M$ satisfying $H(p, x)=h(p)(x) \in$ $M,(p \in P, x \in N)$. 
(ii) We call a mapping $k: X \rightarrow Q$ differentiable if $k$ is a continuous mapping and, for any differentiable mapping $h: P \rightarrow X$ in the sense of (i), the composition $k \circ h: P \rightarrow Q$ is a $C^{\infty}$ mapping between manifolds.

Now, if $\sim$ is an equivalence relation on a mapping space $X$, then we get the quotient space $X / \sim$. Then the canonical projection $\pi=\pi_{X}: X \rightarrow X / \sim$ is defined by $\pi(x)=[x]$ (the equivalence class of $x$ ).

(iii) We call a mapping $\ell: X / \sim \rightarrow Q$ differentiable if the composition $\ell \circ \pi: X \rightarrow Q$ with the projection $\pi$ is differentiable in the sense of (ii).

(iv) We call a mapping $\varphi: X / \sim \rightarrow Y / \approx$ from a mapping space quotient $X / \sim$ to another mapping space quotient $Y / \approx \leftarrow Y \subseteq C^{\infty}(L, K)$ differentiable if $\varphi$ is a continuous mapping and, for any open subset $U \subseteq Y / \approx\left(\leftarrow \pi_{Y}^{-1}(U) \subseteq\right.$ $\left.C^{\infty}(L, K)\right)$ and for any differentiable mapping $\ell: U \rightarrow Q$ in the sense of (iii), the composition $\ell \circ \varphi: \varphi^{-1}(U)\left(\leftarrow \pi_{X}^{-1}\left(\varphi^{-1}(U)\right) \subseteq C^{\infty}(N, M)\right) \rightarrow Q$ is differentiable in the sense of (iii).

(v) We call a mapping $\varphi: X / \sim \rightarrow Y / \approx$ a diffeomorphism if $\varphi$ is differentiable in the sense of (iv) , $\varphi$ is a bijection and the inverse mapping $\varphi^{-1}: Y / \approx \rightarrow X / \sim$ is also differentiable in the sense of (iv). Moreover we call two mapping space quotients $X / \sim$ and $Y / \approx$ diffeomorphic if there exists a diffeomorphism $\varphi: X / \sim$ $\rightarrow Y / \approx$ in the sense of (iv).

Now we give several related results: First, form the definition above, we immediately have that the differentiability is a local notion:

Lemma 2.1 A mapping $\varphi: X / \sim \rightarrow Y / \approx$ is a differentiable mapping if and only if $\varphi$ is locally a differentiable mapping, namely, if and only if, for any $x_{0} \in X / \sim$, there exists a neighborhood $U$ of $x_{0}$ in $X / \sim$, such that $\left.\varphi\right|_{U}: U \rightarrow Y / \approx$ is a differentiable mapping.

Also we observe the followings:

Lemma 2.2 For any $C^{\infty}$ manifold $P, P$ is diffeomorphic to $C^{\infty}(\{\mathrm{pt}\}, P)$.

Proof: In fact the mapping $\varphi: P \rightarrow C^{\infty}(\{\mathrm{pt}\}, P)$ defined by $\varphi(p)(\mathrm{pt})=p,(p \in$ $P)$ is a diffeomorphism.

Example 2.3 Let $\mathbb{Q} \subset \mathbb{R}=C^{\infty}(\mathrm{pt}, \mathbb{R})$ be the set of rational numbers. Then a mapping $h: P \rightarrow \mathbb{Q}$ from a $C^{\infty}$ manifold is differentiable if and only if $h$ is continuous (i.e. locally constant). A mapping $k: \mathbb{Q} \rightarrow Q$ to a $C^{\infty}$ manifold is differentiable if and only if $k$ is continuous.

We show useful lemmata which follow from the definition:

Lemma 2.4 If $h: P \rightarrow X$ is differentiable in the sense of (i), then $\pi \circ h: P \rightarrow$ $X / \sim$ is differentiable in the sense of (iv).

Proof: For any differentiable mapping $\ell: U(\subseteq X / \sim) \rightarrow Q$ in the sense of (iii), the composition $\ell \circ \pi: \pi_{X}^{-1}(U) \rightarrow Q$ is differentiable in the sense of (ii). Therefore $(\ell \circ \pi) \circ h=\ell \circ(\pi \circ h):(\pi \circ h)^{-1}(U) \rightarrow Q$ is differentiable. Hence $\pi \circ h$ differentiable in the sense of (iv). 
Lemma 2.5 The following two conditions are equivalent to each other:

(1) $\varphi: X / \sim \rightarrow Y / \approx$ is differentiable in the sense of (iv).

(2) $\varphi: X / \sim \rightarrow Y / \approx$ is a continuous mapping and, for any differentiable mapping $h: P \rightarrow X$ in the sense of $(\mathrm{i}), \varphi \circ \pi \circ h: P \rightarrow Y / \approx$ is differentiable in the sense of (iv).

Proof: $(1) \Rightarrow(2)$ : Let $\ell: U(\subseteq Y / \approx) \rightarrow Q$ be a differentiable mapping in the sense of (iii). By (1), $\ell \circ(\varphi \circ \pi \circ h)=(\ell \circ \varphi \circ \pi) \circ h:(\varphi \circ \pi \circ h)^{-1}(U) \rightarrow Q$ is differentiable. Therefore $\varphi \circ \pi \circ h: P \rightarrow Y / \approx$ is a differentiable mapping in the sense of (iv).

$(2) \Rightarrow(1)$ : Let $\ell: U(\subseteq Y / \approx) \rightarrow Q$ be differentiable in the sense of (iii), and $h: P \rightarrow(\varphi \circ \pi)^{-1}(U)$ differentiable in the sense of (i). Then $h:(\varphi \circ \pi \circ$ $h)^{-1}(U) \rightarrow(\varphi \circ \pi)^{-1}(U)$ is differentiable in the sense of (i). By (1), we have that $\ell \circ \varphi: \varphi^{-1}(U) \rightarrow Q$ differentiable in the sense of (iii). Therefore we see $(\ell \circ \varphi \circ \pi) \circ h=\ell \circ(\varphi \circ \pi \circ h): P \rightarrow Q$ is $C^{\infty}$. Therefore $\ell \circ \varphi \circ \pi:(\varphi \circ \pi)^{-1}(U) \rightarrow Q$ is differentiable, so is $\ell \circ \varphi: \varphi^{-1}(U) \rightarrow Q$ in the sense of (iii). Hence $\varphi$ is differentiable in the sense of (iv).

Remark 2.6 We remark that, in (2) of Lemma 2.5, we use differentiable functions $h: P \rightarrow X$ to $X$, not to the quotient $X / \sim$, as "test mappings", to teat the differentiability of $\varphi$. Actually, the class of differentiable mappings $P \rightarrow X / \sim$ depends heavily on the nature of the equivalence relation $\sim$.

Lemma 2.7 A differentiable mapping $h: P \rightarrow X \subseteq C^{\infty}(N, M)$ in the sense of (i) is a continuous mapping.

Proof: By the assumption, there exists a differentiable mapping $H: P \times N \rightarrow M$ which satisfies $H(p, x)=h(p)(x)$. Take an open subset of $C^{\infty}(N, M)$ of the form $W(r, K, U)$, where $K \subseteq N$ is a compact subset and $U \subseteq J^{r}(N, M)$ is an open subset.

Suppose, for a $p_{0} \in P, h\left(p_{0}\right)=\left.H\right|_{p_{0} \times N}: N \times M$ belongs to $W(r, K . U)$. Define $j_{1}^{r} H: P \times N \rightarrow J^{r}(N, M)$ by $j_{1}^{r} H(p, x)=j^{r}\left(\left.H\right|_{p \times N}\right)(x)$. Then $j_{1}^{r} H$ is a differentiable mapping in the ordinary sense. In particular it is continuous. From the assumption $h\left(p_{0}\right) \in W(r, K, U),\left(j_{1}^{r} H\right)^{-1}(W(r, K, U))$ is an open neighborhood of $p_{0} \times K$. Since $K$ is compact, there exists an open neighborhood $V$ of $p_{0}$ such that $V \times K \subseteq\left(j_{1}^{r} H\right)^{-1}(W(r, K, U))$. This means that $p_{0} \in V \subseteq h^{-1}(W(r, K, U))$. Therefore $h^{-1}(W(r, K, U))$ is open. Noting that $h^{-1}\left(W(r, K, U) \cap W\left(r^{\prime}, K^{\prime}, U^{\prime}\right)\right)=h^{-1}(W(r, K, U)) \cap h^{-1}\left(W\left(r^{\prime}, K^{\prime}, U^{\prime}\right)\right)$ and that $h^{-1}\left(\cup W_{\nu}\right)=\cup h^{-1}\left(W_{\nu}\right)$, we see that $h$ is continuous.

Remark 2.8 (The reason we adopt the $C^{\infty}$ topology.) Lemma 2.7 does not hold for the Whitney $C^{\infty}$ topology. For example, in $X=C^{\infty}(\mathbb{R}, \mathbb{R})$, consider the differentiable mapping $h: \mathbb{R} \rightarrow C^{\infty}(\mathbb{R}, \mathbb{R})$ defined by the differentiable mapping $H(\lambda, x):=\lambda$. Then $h(0)$ is identically 0 . Its graph is $\mathbb{R} \times 0 \subset \mathbb{R} \times \mathbb{R}$. Then there exists an open set $U$ containing $\mathbb{R} \times 0$ such that $h^{-1}(W(U))=\{0\}$. Then $W(U)$ is an open subset of $C^{\infty}(\mathbb{R}, \mathbb{R})$ with respect to the Whitney $C^{\infty}$ topology, while $h^{-1}(W(U))=\{0\} \subset \mathbb{R}$ is not open in $\mathbb{R}$. Therefore $h$ is not continuous in the Whitney $C^{\infty}$ topology. 
Remark 2.9 (The continuity condition in (ii) is necessary.) In the above definition (ii), the continuity of $k$ is not implied from just the condition that for any differentiable mapping $h: P \rightarrow X$ in the sense (i), the composition $k \circ h: P \rightarrow Q$ is differentiable.

In fact set $X=\{1 / n\} \cup\{0\} \subset \mathbb{R}=C^{\infty}(\{$ pt $\}, \mathbb{R})$ and $Y=\{0,1\}=$ $C^{\infty}(\{\mathrm{pt}\},\{0,1\})$.

Define $k: X \rightarrow Y$ by $k(1 / n)=1, k(0)=0$. Then any differentiable mapping $h: P \rightarrow X$ is locally constant, and so is $k \circ h: P \rightarrow Y$. Then $k \circ h$ is differentiable, while $k$ is not continuous.

Thus, in the definition (ii), we need the continuity of $k$.

Lemma 2.10 (1) The identity mapping id $: X / \sim \rightarrow X / \sim$ is differentiable. (2) Let $\varphi: X / \sim \rightarrow Y / \approx$ and $\psi: Y / \approx \rightarrow Z / \equiv$ be differentiable mappings. Then the composition $\psi \circ \varphi: X / \sim \rightarrow Z / \equiv$ is differentiable.

Proof: (1) is clear since id is continuous. (2) Since $\varphi$ and $\psi$ are continuous, $\psi \circ \varphi$ is continuous. Let $\ell: U(\subseteq Z / \equiv) \rightarrow Q$ be a differentiable mapping. Then $\ell \circ \psi$ is differentiable by (iv). By (iv) again, $(\ell \circ \psi) \circ \varphi=\ell \circ(\psi \circ \varphi)$ is differentiable. Hence $\psi \circ \varphi$ is differentiable.

Lemma 2.11 (1) The quotient mapping $\pi: X \rightarrow X / \sim$ is differentiable. (2) A mapping $\varphi: X / \sim \rightarrow Y / \approx$ is differentiable if and only if $\varphi \circ \pi: X \rightarrow Y / \approx i s$ differentiable.

Proof: (1) First $\pi$ is continuous by the definition of the quotient topology. Let $\ell: U(\subseteq X / \sim) \rightarrow Q$ be a differentiable mapping. Then $\ell \circ \pi: \pi^{-1}(U) \rightarrow Q$ is differentiable by the definition (iii). Therefore, by the definition (iv), $\pi$ is differentiable.

(2) First note that $\varphi$ is continuous if and only if $\varphi \circ \pi$ is continuous. If $\varphi$ is differentiable, then $\varphi \circ \pi$ is differentiable by (1) and Lemma 2.10. Conversely let $\varphi \circ \pi$ be differentiable, and $\ell: U(\subseteq Y / \approx) \rightarrow Q$ be differentiable. Then $\ell \circ(\varphi \circ \pi)=(\ell \circ \varphi) \circ \pi:(\ell \circ \varphi \circ \pi)^{-1}(U) \rightarrow Q$ is differentiable. Therefore $\ell \circ \varphi:(\ell \circ \varphi)^{-1}(U) \rightarrow Q$ is differentiable. Thus, by (iv), $\varphi$ is differentiable.

Remark 2.12 Let $X / \sim$ be a mapping space quotient and $U \subseteq X / \sim$ be an open subset. Then

$$
E(U):=\{\ell: U \rightarrow \mathbb{R} \mid \ell \text { is differentiable. }\}
$$

is an $\mathbb{R}$-algebra. In fact, for $\ell, \ell^{\prime} \in E(U)$, we have $\ell+\ell^{\prime}, \ell \cdot \ell^{\prime} \in E(U)$. Moreover any constant function $U \rightarrow \mathbb{R}$ is differentiable. Furthermore, for any $\ell_{1}, \ldots, \ell_{r} \in$ $E(U)$ and for any $C^{\infty}$ function $\tau: \mathbb{R}^{r} \rightarrow \mathbb{R}$, the composition $\tau \circ\left(\ell_{1}, \ldots, \ell_{r}\right)$ belongs to $E(U)$. Thus $E(U)$ is a $C^{\infty}$-ring and $E$ is a sheaf of $C^{\infty}$-rings in the sense of [15].

We have the following results: 
Lemma 2.13 For $C^{\infty}$ manifolds $N, M, L$, the composition $c: C^{\infty}(N, M) \times$ $C^{\infty}(M, L) \rightarrow C^{\infty}(N, L)$ is differentiable.

Proof: First we remark that $c$ is continuous for the $C^{\infty}$ topology [9]. Let $h$ : $P \rightarrow C^{\infty}(N, M) \times C^{\infty}(M, L)$ be a differentiable mapping. Suppose $H: P \times$ $(N \amalg M)=P \times N \amalg P \times M \rightarrow(M \amalg L)$ is a $C^{\infty}$ mapping defining $h$. Set $h(p)=(f(p), g(p)), p \in P$. Then $H(P \times N) \subseteq M$ and $H(P \times M) \subseteq L$. Then $f(p)(x)=H(p, x)$ for $p \in P, x \in X$, and $g(p)(y)=H(p, y)$ for $p \in P, y \in Y$. Then $(c \circ h)(p)(x)=g(p)(f(p)(x))=H(p, H(p, x))$. Therefore the $C^{\infty}$ mapping $K: P \times N \rightarrow L$ defined by $K(p, x):=H(p, H(p, x))$ defines $c \times h$. Therefore $c \circ h$ is differentiable. Therefore, by Lemma 2.5, we see $c$ is differentiable.

Lemma 2.14 If $N$ and $N^{\prime}$ are diffeomorphic, and if $M$ and $M^{\prime}$ are diffeomorphic, then $C^{\infty}(N, M)$ and $C^{\infty}\left(N^{\prime}, M^{\prime}\right)$ are diffeomorphic.

Proof: Let $\sigma: N \rightarrow N^{\prime}$ and $\tau: M \rightarrow M^{\prime}$ be diffeomorphisms. Then set $\varphi: C^{\infty}(N, M) \rightarrow C^{\infty}\left(N^{\prime}, M^{\prime}\right)$ by $\varphi(f):=\tau \circ f \circ \sigma^{-1}$. Then $\varphi$ is differentiable. In fact, let $h: P \rightarrow C^{\infty}(N, M)$ be a differentiable mapping. Suppose $h$ is defined by a $C^{\infty}$ mapping $H: P \times N \rightarrow M$. Then $\varphi \circ h: P \rightarrow C^{\infty}\left(N^{\prime}, M^{\prime}\right)$ is defined by the $C^{\infty}$ mapping $\tau \circ H \circ\left(\operatorname{id}_{P} \times \sigma^{-1}\right): P \times N^{\prime} \rightarrow M^{\prime}$. Therefore $\varphi \circ h$ is differentiable. By Lemma 2.5, we see $\varphi$ is differentiable. By symmetry, define $\psi: C^{\infty}\left(N^{\prime}, M^{\prime}\right) \rightarrow C^{\infty}(N, M)$ by $\psi(g):=\tau^{-1} \circ g \circ \sigma$. Then $\psi$ is differentiable and it is the inverse of $\varphi$. Therefore $\varphi$ is a diffeomorphism.

\section{Mild quotients.}

Apart from general setting, we select "mild quotients "among general mapping space quotients in our purpose.

A mapping space $X \subseteq C^{\infty}(N, M)$ is called a mild space

(a): the topology of $X$ has a countable basis, and

(b): for any $x_{0} \in X$ and for any sequence $x_{n} \in X,(n=1,2,3, \ldots)$ with $x_{n} \rightarrow$ $x_{0}(n \rightarrow \infty)$, there exist a differentiable mapping $h: \mathbb{R} \rightarrow X$, a subsequence $x_{n_{k}}$ and a sequence $p_{0}, p_{k} \in \mathbb{R}(k=1,2,3, \ldots)$ satisfying $p_{k} \rightarrow p_{0}(k \rightarrow \infty)$ and $h\left(p_{k}\right)=x_{n_{k}}(k=1,2,3, \ldots)$.

A mapping space quotient is called a mild quotient if, it is diffeomorphic to a quotient $X / \sim$ of a mild space $X$.

The motivation of introducing the notion of mild quotient lies in the following (cf. Lemma 2.5):

Lemma 3.1 Let $\varphi: X / \sim \rightarrow Z / \equiv$ be a mapping between mapping space quotients. Suppose that $X / \sim$ is a mild quotient of a mild space $X$ and that $\varphi$ satisfying the condition: For any differentiable mapping $h: P \rightarrow X$ from a differentiable manifold $P$, the composition $\varphi \pi \circ \circ h: P \rightarrow Z / \equiv$ is differentiable. Then $\varphi$ is a continuous mapping. Therefore $\varphi$ is differentiable by Lemma 2.5. 
Proof: Assume $\varphi$ is not continuous. Then $\varphi \circ \pi: X \rightarrow Z / \equiv$ is not continuous. Note that $\varphi: X / \sim \rightarrow Z / \equiv$ is continuous if and only if $\varphi \circ \pi: X \rightarrow Z / \equiv$ is continuous. We may suppose $X$ itself is a mild space, i.e., $X$ satisfies the conditions (a)(b). By (a), $X$ has the topology with countable bases. Then there exist a point $x_{0} \in X$ and a sequence $x_{n} \in X$ with $x_{n} \rightarrow x_{0}(n \rightarrow \infty)$ while the sequence $(\varphi \circ \pi)\left(x_{n}\right)$ does not converge to $(\varphi \circ \pi)\left(x_{0}\right) \in Y / \approx$; there exists an open set $V \subset Y / \approx$ with $(\varphi \circ \pi)\left(x_{0}\right) \in V,(\varphi \circ \pi)\left(x_{n}\right) \notin V,(n=1,2,3, \ldots)$. Since $X$ is a mild space, there exist a differential mapping $h: \mathbb{R} \rightarrow X$, a subsequence $x_{n_{k}}$ and a sequence $p_{k} \in \mathbb{R}$ with $p_{k} \rightarrow p_{0} \in P$ and $h\left(p_{k}\right)=x_{n_{k}}$. Then $(\varphi \circ \pi) \circ h: P \rightarrow Y / \approx$ must be continuous. Therefore $((\varphi \circ \pi) \circ h)\left(p_{k}\right)=$ $(\varphi \circ \pi)\left(x_{n_{k}}\right)$ converges to $((\varphi \circ \pi) \circ h)\left(p_{0}\right)=(\varphi \circ \pi)\left(h\left(p_{0}\right)\right)=(\varphi \circ \pi)\left(x_{0}\right)$. This leads to a contradiction.

Example 3.2 A $C^{\infty}$ manifold is a mild space.

Example 3.3 ([20]) Let $N, M$ be differentiable manifolds. Then $C^{\infty}(N, M)$ is a mild space. In fact let $f_{n} \in C^{\infty}(N, M),(n=1,2,3, \ldots)$ be a sequence converging to $f_{0} \in C^{\infty}(N, M)$ for the $C^{\infty}$ topology. Then, using Hestence's lemma [28], we see that there exist a positive sequence $a_{k}$, a subsequence $f_{n_{k}}$ of $f_{n}$, a $C^{\infty}$ mapping $H: \mathbb{R} \times N \rightarrow M$ satisfying $a_{k} \rightarrow 0$ and $H\left(a_{k}, x\right)=$ $f_{n_{k}}(x),(x \in N, n=1,2,3, \ldots)$. Then we have the differentiable mapping $h$ : $\mathbb{R} \rightarrow C^{\infty}(N, M)$ defined by $h(t)(x)=H(t, x),(t \in \mathbb{R}, x \in X)$ and that $h\left(a_{k}\right)=$ $f_{n_{k}}$ with $a_{k} \rightarrow 0$.

Example 3.4 Let $M$ be a manifold. Let $\operatorname{Diff}(M)\left(\operatorname{resp} \operatorname{Diff}_{c}(M)\right)$ denotes the space of all diffeomorphisms (resp. diffeomorphisms with compact supports). Then $\operatorname{Diff}_{c}(M)$ is a mild space. (I do not know whether $\operatorname{Diff}(M)$ is mild or not if $M$ is non-compact. )

Example 3.5 The subspace $\mathbb{Q} \subset \mathbb{R}$, the set of rational numbers in the real, is not mild.

Example 3.6 Open subsets of a mild quotient are mild: Let $\bar{X}=X / \sim$ be a quotient space of of a mild space $X \subset C^{\infty}(N, M)$ by the projection $\pi: X \rightarrow \bar{X}$. Let $W \subset \bar{X}$ be an open subset of $\bar{X}$. Set $X^{\prime}:=\pi^{-1}(W)$. Then $W$ is regarded as a quotient space by $\pi: X^{\prime} \rightarrow W$. Then $W$ is a mild space.

The following is easy to verify:

Lemma 3.7 Let $X \subseteq C^{\infty}(N, M)$ and $Y \subseteq C^{\infty}(L, \Lambda)$ be mild spaces. Then the product $X \times Y \subseteq C^{\infty}(N \amalg L, M \amalg \Lambda)$ is a mild space.

\section{Differentiable structure on a moduli space.}

Recall that we have defined the labelled moduli space of $f: S^{1} \rightarrow \mathbb{R}^{2}$ as the mapping space quotient

$$
\widetilde{\mathcal{M}}_{\text {symp }}(f)=\operatorname{Diff}^{+}\left(\mathbb{R}^{2}\right) / \sim_{f},
$$


of $\operatorname{Diff}^{+}\left(\mathbb{R}^{2}\right) \subset C^{\infty}\left(\mathbb{R}^{2}, \mathbb{R}^{2}\right)$. Therefore naturally we define the differentiable structure on $\widetilde{\mathcal{M}}_{\text {symp }}(f)$. Also we can consider another mapping space quotient $\operatorname{Diff}_{c}\left(\mathbb{R}^{2}\right) / \sim_{f}$ of $\operatorname{Diff}_{c}\left(\mathbb{R}^{2}\right) \subset \operatorname{Diff}^{+}\left(\mathbb{R}^{2}\right) \subset C^{\infty}\left(\mathbb{R}^{2}, \mathbb{R}^{2}\right)$. In fact we have the following:

Lemma 4.1 $\operatorname{Diff}^{+}\left(\mathbb{R}^{2}\right) / \sim_{f}$ is diffeomorphic to $\operatorname{Diff}_{c}\left(\mathbb{R}^{2}\right) / \sim_{f}$.

Proof: Let $D_{1}=D(R) \subset \mathbb{R}^{2}$ be a closed disk with radius $R$ whose interior contains $f\left(S^{1}\right)$. Set $D_{2}=D(2 R)$. We denote by $\operatorname{Diff}_{D}\left(\mathbb{R}^{2}\right)$ the set of diffeomorphisms on $\mathbb{R}^{2}$ with support contained in $D$. Then we show that

$$
\operatorname{Diff}^{+}\left(\mathbb{R}^{2}\right) / \sim_{f} \cong \operatorname{Diff}_{D_{2}}\left(\mathbb{R}^{2}\right) / \sim_{f} \cong \operatorname{Diff}_{c}\left(\mathbb{R}^{2}\right) / \sim_{f}
$$

Denote by $i: \operatorname{Diff}_{D_{2}}\left(\mathbb{R}^{2}\right) \rightarrow \operatorname{Diff}^{+}\left(\mathbb{R}^{2}\right)$ the inclusion. Then $i$ induces the mapping $\varphi: \operatorname{Diff}_{D_{2}}\left(\mathbb{R}^{2}\right) / \sim_{f} \rightarrow \operatorname{Diff}^{+}\left(\mathbb{R}^{2}\right) / \sim_{f}$ which is differentiable and bijective. In fact we define the inverse $\psi: \operatorname{Diff}^{+}\left(\mathbb{R}^{2}\right) / \sim_{f} \rightarrow \operatorname{Diff}_{D_{2}}\left(\mathbb{R}^{2}\right) / \sim_{f}$ by using

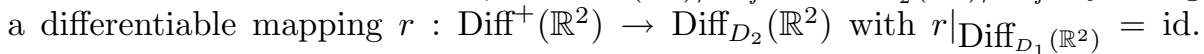
Therefore $\varphi$ is a diffeomorphism. A diffeomorphism between Diff ${ }_{c}\left(\mathbb{R}^{2}\right) / \sim_{f}$ and $\operatorname{Diff}_{D_{2}}\left(\mathbb{R}^{2}\right) / \sim_{f}$ is obtained similarly.

Recall that the symplectic moduli space has been defined by $\mathcal{M}_{\text {symp }}(f)=$ $\operatorname{Diff}^{+}\left(\mathbb{R}^{2}\right) f / \operatorname{Symp}\left(\mathbb{R}^{2}\right)$. Therefore it is regarded as a quotient of a subspace of $C^{\infty}\left(S^{1}, \mathbb{R}^{2}\right)$. In fact Diff $\left(\mathbb{R}^{2}\right) f \subset C^{\infty}\left(S^{1}, \mathbb{R}^{2}\right) / \operatorname{Diff}^{+}\left(S^{1}\right)$. Note that $f \in$ $C^{\infty}\left(S^{1}, \mathbb{R}^{2}\right) / \operatorname{Diff}^{+}\left(S^{1}\right)$. On the other hand, since there is a canonical surjection $\Pi: \widetilde{\mathcal{M}}_{\text {symp }}(f) \rightarrow \mathcal{M}_{\text {symp }}(f)$ defined by $\Pi([\rho])=[\rho \circ f]$, the space $\mathcal{M}_{\text {symp }}(f)$ can be regarded as a quotient of $\operatorname{Diff}^{+}\left(\mathbb{R}^{2}\right) \subset C^{\infty}\left(\mathbb{R}^{2}, \mathbb{R}^{2}\right)$ as well. However the differentiable structure of $\mathcal{M}_{\text {symp }}(f)$ does not depend on these representations as mapping space quotients as we will see below.

We have set, for an oriented curve $f \in C^{\infty}\left(S^{1}, \mathbb{R}^{2}\right) / \operatorname{Diff}^{+}\left(S^{1}\right)$, the symmetry group of $f$,

$$
S_{f}:=\left\{\rho \in \operatorname{Diff}^{+}\left(\mathbb{R}^{2}\right) \mid \rho \circ f=f \bmod . \operatorname{Diff}^{+}\left(S^{1}\right)\right\} .
$$

Moreover we set, the groups of symmetries with compact supports,

$$
S_{c, f}:=\left\{\rho \in \operatorname{Diff}_{c}\left(\mathbb{R}^{2}\right) \mid \rho \circ f=f \bmod . \operatorname{Diff}^{+}\left(S^{1}\right)\right\} .
$$

Then, in fact we have the following:

Lemma 4.2 If $f$ is of finite type, then there are diffeomorphisms

$$
\begin{array}{r}
\operatorname{Diff}_{c}\left(\mathbb{R}^{2}\right) / S_{c, f} \cong \operatorname{Diff}^{+}\left(\mathbb{R}^{2}\right) / S_{f} \cong \operatorname{Diff}^{+}\left(\mathbb{R}^{2}\right) f \\
\left(=\operatorname{Diff}_{c}\left(\mathbb{R}^{2}\right) f \subset C^{\infty}\left(S^{1}, \mathbb{R}^{2}\right) / \operatorname{Diff}^{+}\left(S^{1}\right)\right)
\end{array}
$$

Proof: The first diffeomorphism is given similarly as in Lemma 4.1. To give the second diffeomorphism define $\widetilde{\varphi}: \operatorname{Diff}^{+}\left(\mathbb{R}^{2}\right) \rightarrow \operatorname{Diff}^{+}\left(\mathbb{R}^{2}\right) f$ by $\varphi(\rho)=\rho \circ f$. Then 
$\varphi$ is differentiable and $\varphi$ induces a differentiable mapping $\varphi: \operatorname{Diff}^{+}\left(\mathbb{R}^{2}\right) / S_{f} \rightarrow$ $\operatorname{Diff}^{+}\left(\mathbb{R}^{2}\right) f$, which is bijective. To see the inverse of $\varphi$ is differentiable, we take any differentiable mapping $h: P \rightarrow C^{\infty}\left(S^{1}, \mathbb{R}^{2}\right)$ which induces a differentiable mapping $\bar{h}: P \rightarrow \operatorname{Diff}^{+}\left(\mathbb{R}^{2}\right) f$, and we show that there exists a differentiable mapping $\widetilde{h}: P \rightarrow \operatorname{Diff}^{+}\left(\mathbb{R}^{2}\right)$ that $\widetilde{\varphi} \circ \widetilde{h}=\bar{h}$. To do that, for a finite dimensional $C^{\infty}$ family $f_{\lambda}$ isotopic to $f, \lambda \in P$, we have to find a finite dimensional $C^{\infty}$ family of diffeomorphisms $\rho_{\lambda}$ on $\mathbb{R}^{2}$ and $\sigma_{\lambda}$ on $S^{1}$ such that $\rho_{\lambda} \circ f \circ \sigma_{\lambda}=f_{\lambda}$. (An isotopy is covered by an ambient isotopy). Since $f$ is of finite type, any multi-germ of $f$ is finitely determined([24][31]). Therefore, any $C^{\infty}$ family in an orbit is covered by a $C^{\infty}$ family of diffeomorphisms locally at any point on $f\left(S^{1}\right)$. By patching local ambient isotopies, we find a global ambient isotopy $\left(\rho_{\lambda}, \sigma_{\lambda}\right)$.

Thus we have the following basic result:

Lemma 4.3 Let $f \in C^{\infty}\left(S^{1}, \mathbb{R}^{2}\right) / \operatorname{Diff}^{+}\left(S^{1}\right)$ be an oriented curve. Then the labelled symplectic moduli space $\widetilde{\mathcal{M}}_{\text {symp }}(f)=\operatorname{Diff}^{+}\left(\mathbb{R}^{2}\right) / \sim_{f}$ is a mild quotient. If $f: S^{1} \rightarrow \mathbb{R}^{2}$ is of finite type, then $\operatorname{Diff}^{+}\left(\mathbb{R}^{2}\right) f \subseteq C^{\infty}\left(S^{1}, \mathbb{R}^{2}\right) / \operatorname{Diff}^{+}\left(S^{1}\right)$ is a mild quotient. Therefore $\mathcal{M}_{\text {symp }}(f)=\operatorname{Diff}^{+}\left(\mathbb{R}^{2}\right) f / \operatorname{Symp}\left(\mathbb{R}^{2}\right)$ is a mild quotient.

Remark 4.4 The space of germs $C^{\infty}\left((N, S),\left(M, y_{0}\right)\right)$ is a mild quotient, since it is a quotient of the mild space $C^{\infty}(N, M)$. Similarly to Lemma 4.3, we see also the (labelled) local symplectic moduli space of a map-germ of finite codimension is a mild quotient.

\section{The space of symplectic forms.}

Differential two-forms $\omega, \omega^{\prime}$ on $\mathbb{R}^{2}$ are equivalent if there exists a diffeomorphism $\rho$ on $\mathbb{R}^{2}$ such that $\omega=\rho^{*} \omega^{\prime}$. Let $\mathcal{S}\left(\mathbb{R}^{2}\right)$ denote the space of symplectic forms on $\mathbb{R}^{2}$ which are equivalent to the standard symplectic form $\omega_{0}=d x \wedge d y$. It is known that $\omega \in \mathcal{S}\left(\mathbb{R}^{2}\right)$ if and only if $\int_{\mathbb{R}^{2}} \omega= \pm \infty$ (cf. [10]). Note that $\mathcal{S}\left(\mathbb{R}^{2}\right)$ is a subset of $C^{\infty}\left(\mathbb{R}^{2}, \bigwedge^{2}\left(T^{*} \mathbb{R}^{2}\right)\right)$. A symplectic form on $\mathbb{R}^{2}$ is called positive if it gives the standard orientation on $\mathbb{R}^{2}$. Then also consider the subspace $\mathcal{S}^{+}\left(\mathbb{R}^{2}\right) \subset \mathcal{S}\left(\mathbb{R}^{2}\right)$ consisting of positive forms on $\mathbb{R}^{2}$. Note that the standard symplectic form $\omega_{0}=d x \wedge d y$ belongs to $\mathcal{S}^{+}\left(\mathbb{R}^{2}\right)$.

Actually we treat another object $\mathcal{S}_{c}\left(\mathbb{R}^{2}\right)$, the space of symplectic forms on $\mathbb{R}^{2}$ with "compact support", namely symplectic forms which agree with $\omega_{0}$ outside of compact subsets. Note that $\mathcal{S}_{c}\left(\mathbb{R}^{2}\right) \subset \mathcal{S}^{+}\left(\mathbb{R}^{2}\right)$. Moreover we see that, for any $\omega \in \mathcal{S}_{c}\left(\mathbb{R}^{2}\right)$, there exists a $\rho \in \operatorname{Diff}\left(\mathbb{R}^{2}\right)$ with compact support satisfying $\omega=\rho^{*} \omega_{0}$. We consider the group $\operatorname{Diff}_{c}\left(\mathbb{R}^{2}\right)\left(\operatorname{resp} . \operatorname{Symp}_{c}\left(\mathbb{R}^{2}\right)\right.$ ) of diffeomorphisms (resp. symplectomorphisms) on $\mathbb{R}^{2}$ with compact supports. We define an equivalence relation $\sim$ on $\operatorname{Diff}_{c}\left(\mathbb{R}^{2}\right)$, using the subgroup $\operatorname{Symp}_{c}\left(\mathbb{R}^{2}\right)$; $\rho_{1}, \rho_{2} \in \operatorname{Diff}_{c}\left(\mathbb{R}^{2}\right)$ are equivalent, $\rho_{1} \sim \rho_{2}$, if $\rho_{1}=\tau \circ \rho_{2}$ for some $\tau \in \operatorname{Symp}_{c}\left(\mathbb{R}^{2}\right)$. 
Then we have the quotient space $\operatorname{Diff}_{c}\left(\mathbb{R}^{2}\right) / \sim$. Though the action is a left action, we denote the quotient space by $\operatorname{Diff}_{c}\left(\mathbb{R}^{2}\right) / \operatorname{Symp}_{c}\left(\mathbb{R}^{2}\right)$. Recall that the notion of mild spaces introduced in $\S 3$. Then, similarly to Example 3.4, we have:

Lemma 5.1 $\mathcal{S}_{c}\left(\mathbb{R}^{2}\right) \subset C^{\infty}\left(\mathbb{R}^{2}, \bigwedge^{2}\left(T^{*} \mathbb{R}^{2}\right)\right)$ is a mild space. Therefore any quotient space of $\mathcal{S}_{c}\left(\mathbb{R}^{2}\right)$ is a mild quotient.

Moreover we have:

Lemma $5.2 \mathcal{S}_{c}\left(\mathbb{R}^{2}\right)$ is diffeomorphic to $\operatorname{Diff}_{c}\left(\mathbb{R}^{2}\right) / \operatorname{Symp}_{c}\left(\mathbb{R}^{2}\right)$.

Proof: Define a mapping $\Phi: \operatorname{Diff}_{c}\left(\mathbb{R}^{2}\right) \rightarrow \mathcal{S}_{c}\left(\mathbb{R}^{2}\right)$ by $\Phi(\rho)=\rho^{*} \omega_{0}$. Then $\Phi$ is a surjective mapping. Note that if $\rho_{1}=\tau \circ \rho_{2}$ with $\tau \in \operatorname{Symp}_{c}\left(\mathbb{R}^{2}\right)$, then $\rho_{1}^{*} \omega_{0}=\rho_{2}^{*} \omega_{0}$, therefore $\rho_{1} \circ \rho_{2}^{-1} \in \operatorname{Symp}_{c}\left(\mathbb{R}^{2}\right)$. The mapping $\Phi$ induces a mapping $\varphi: \operatorname{Diff}_{c}\left(\mathbb{R}^{2}\right) / \operatorname{Symp}_{c}\left(\mathbb{R}^{2}\right) \rightarrow \mathcal{S}_{c}\left(\mathbb{R}^{2}\right)$ defined by $\varphi([\rho])=\rho^{*} \omega_{0}$. Then $\varphi$ is a bijection. Let $h: P \rightarrow \operatorname{Diff}_{c}\left(\mathbb{R}^{2}\right)$ be any differentiable mapping from any manifold. Let $H: P \times \mathbb{R}^{2} \rightarrow \mathbb{R}^{2}$ be a $C^{\infty}$ mapping which defines $h$. Then we set $H^{\prime}: P \times \mathbb{R}^{2} \rightarrow \bigwedge^{2}\left(T^{*} \mathbb{R}^{2}\right)$ by $H^{\prime}(p, x, y)=\left(h(p)^{*} \omega\right)(x, y)$. Then $H^{\prime}$ is $C^{\infty}$ and defines $\Phi \circ h$. Therefore $\Phi \circ h$ is differentiable. Since Diff $c\left(\mathbb{R}^{2}\right)$ is a mild space, we see $\Phi$ is differentiable by Lemma 2.5. Therefore $\phi$ is differentiable.

Conversely define $\widetilde{\psi}: \mathcal{S}_{c}\left(\mathbb{R}^{2}\right) \rightarrow \operatorname{Diff}_{c}\left(\mathbb{R}^{2}\right)$ by

$$
\widetilde{\psi}(\omega)(x, y):=\left(\int_{0}^{x} f(x, y) d x, y\right)
$$

where $\omega=f(x, y) d x \wedge d y$. Then $\widetilde{\psi}$ induces a mapping

$$
\psi: \mathcal{S}_{c}\left(\mathbb{R}^{2}\right) \rightarrow \operatorname{Diff}_{c}\left(\mathbb{R}^{2}\right) / \operatorname{Symp}_{c}\left(\mathbb{R}^{2}\right) .
$$

We see that $\psi$ is the inverse of $\varphi$. To show $\psi$ is differentiable, let $k: P \rightarrow \mathcal{S}_{c}\left(\mathbb{R}^{2}\right)$ be a differentiable mapping. Let $K: P \times \mathbb{R}^{2} \rightarrow \bigwedge^{2}\left(T^{*} \mathbb{R}^{2}\right)$ be a $C^{\infty}$ mapping which defines $k$. Set

$$
K^{\prime}(p, x, y)=\left(\int_{0}^{x} f(p, x, y) d x, y\right)
$$

where $K^{\prime}(p, x, y)=f(p, x, y) d x \wedge d y$. Then $K^{\prime}: P \times \mathbb{R}^{2} \rightarrow \mathbb{R}^{2}$ is $C^{\infty}$ and $K^{\prime}$ defines $\widetilde{\psi} \circ k: P \rightarrow \operatorname{Diff}_{c}\left(\mathbb{R}^{2}\right)$. Therefore $\widetilde{\psi} \circ k$ is differentiable. Thus $\psi \circ k=\pi \circ(\widetilde{\psi} \circ k): P \rightarrow \operatorname{Diff}_{c}\left(\mathbb{R}^{2}\right) / \operatorname{Symp}_{c}\left(\mathbb{R}^{2}\right)$ is differentiable. Since $\mathcal{S}_{c}\left(\mathbb{R}^{2}\right)$ is a mild space, we see $\psi$ is continuous. Therefore $\psi$ is differentiable, by Lemma 2.5. Thus we show that $\varphi$ is a diffeomorphism.

For a oriented plane curve $f \in C^{\infty}\left(S^{1}, \mathbb{R}^{2}\right) / \operatorname{Diff}^{+}\left(S^{1}\right)$, we have considered the group of symmetry of $f$ :

$$
S_{f}:=\left\{\rho \in \operatorname{Diff}^{+}\left(\mathbb{R}^{2}\right) \mid \rho \circ f=f \text { up to } \operatorname{Diff}^{+}\left(S^{1}\right)\right\} .
$$


Furthermore, if $f$ is labelled, then we consider the group of label-preserving symmetry of $f$ :

$$
\underline{S}_{f}:=\left\{\rho \in S_{f} \mid \rho \text { preserves the labelling of } f .\right\} .
$$

Moreover set $S_{c, f}:=S_{f} \cap \operatorname{Diff} c\left(\mathbb{R}^{2}\right)$ and $\underline{S}_{c, f}:=\underline{S}_{f} \cap \operatorname{Diff}_{c}\left(\mathbb{R}^{2}\right)$.

Note that there exist exact sequences of groups:

$$
1 \rightarrow \underline{S}_{f} \rightarrow S_{f} \rightarrow G_{f} \rightarrow 1, \quad 1 \rightarrow \underline{S}_{c, f} \rightarrow S_{c, f} \rightarrow G_{f} \rightarrow 1 .
$$

On the other hand, let $\omega \in \mathcal{S}^{+}\left(\mathbb{R}^{2}\right)$. Then there exists $\rho \in \operatorname{Diff}^{+}\left(\mathbb{R}^{2}\right)$ such that $\omega=\rho^{*} \omega_{0}$. If $\rho_{1}^{*} \omega_{0}=\rho_{2}^{*} \omega_{0}$, then $\tau:=\rho_{1} \circ \rho_{2}^{-1} \in \operatorname{Symp}\left(\mathbb{R}^{2}\right)$, and $\tau \circ \rho_{2}=\rho_{1}$, therefore, $\tau \circ \rho_{2} \circ f=\rho_{1} \circ f$. Thus a mapping

$$
p: \mathcal{S}^{+}\left(\mathbb{R}^{2}\right) \rightarrow \widetilde{\mathcal{M}}_{\text {symp }}(f)
$$

is well-defined by $p(\omega):=[\rho] \in \operatorname{Diff}^{+}\left(\mathbb{R}^{2}\right) / \sim_{f}$ for some $\rho \in \operatorname{Diff}^{+}\left(\mathbb{R}^{2}\right)$ with $\omega=\rho^{*} \omega_{0}$. Moreover we have the diagram:

$$
\begin{aligned}
& \operatorname{Diff}_{c}\left(\mathbb{R}^{2}\right) \stackrel{i}{\rightarrow} \operatorname{Diff}^{+}\left(\mathbb{R}^{2}\right) \quad \stackrel{\Pi}{\rightarrow} \quad \widetilde{\mathcal{M}}_{\text {symp }}(f) \stackrel{\stackrel{\pi}{\rightarrow}}{\mathcal{M}_{\text {symp }}(f)} \\
& \Phi \downarrow \quad \Phi \downarrow \quad \nearrow p \\
& \mathcal{S}_{c}\left(\mathbb{R}^{2}\right) \stackrel{j}{\rightarrow} \mathcal{S}^{+}\left(\mathbb{R}^{2}\right)
\end{aligned}
$$

Here $i$ and $j$ are inclusions, $\Pi$ and $\pi$ are projections. We set $\Phi(\rho)=\rho^{*} \omega_{0}$, for $\omega \in \operatorname{Diff}^{+}\left(\mathbb{R}^{2}\right)$.

Then we have

Lemma 5.3 (1) There are diffeomorphisms

$$
\begin{gathered}
\mathcal{S}^{+}\left(\mathbb{R}^{2}\right) / S_{f} \cong \mathcal{S}_{c}\left(\mathbb{R}^{2}\right) / S_{c, f} \cong \operatorname{Diff}_{c}\left(\mathbb{R}^{2}\right) f / \operatorname{Symp}_{c}\left(\mathbb{R}^{2}\right) \\
\cong \operatorname{Diff}^{+}\left(\mathbb{R}^{2}\right) f / \operatorname{Symp}\left(\mathbb{R}^{2}\right)=: \mathcal{M}_{\text {symp }}(f) .
\end{gathered}
$$

(2) There are diffeomorphisms

$$
\mathcal{S}^{+}\left(\mathbb{R}^{2}\right) / \underline{S}_{f} \cong \mathcal{S}_{c}\left(\mathbb{R}^{2}\right) / \underline{S}_{c, f} \cong \operatorname{Diff}_{c}\left(\mathbb{R}^{2}\right) / \sim_{f} \cong \operatorname{Diff}^{+}\left(\mathbb{R}^{2}\right) / \sim_{f}=: \widetilde{\mathcal{M}}_{\text {symp }}(f) .
$$

Proof: For $\omega, \omega^{\prime} \in \mathcal{S}_{c}\left(\mathbb{R}^{2}\right)$, suppose $p \circ j\left(\omega_{1}\right)=p \circ j\left(\omega_{2}\right)$. Then $\omega_{1}=\rho_{1}^{*} \omega_{0}, \omega_{2}=$ $\rho_{2}^{*} \omega_{0}$ and $\tau \circ \rho_{1} \circ f=\rho_{2} \circ f$ for some $\rho_{1}, \rho_{2} \in \operatorname{Diff}_{c}\left(\mathbb{R}^{2}\right), \tau \in \operatorname{Symp}_{c}\left(\mathbb{R}^{2}\right), \tau$ being label-preserving. Then $\rho_{2}^{-1} \circ \tau \circ \rho_{1} \in \underline{S}_{c, f}$ and $\left(\rho_{2}^{-1} \circ \tau \circ \rho_{1}\right)^{*} \omega_{2}=\omega_{1}$. Conversely suppose $\rho^{*} \omega_{2}=\omega_{1}$, for some $\rho \in \underline{S}_{c, f}$. Then $\rho_{2} \circ \rho \circ \rho_{1}^{-1}=: \tau \in \operatorname{Symp}_{c}\left(\mathbb{R}^{2}\right)$. Since $\rho=\rho_{2}^{-1} \circ \tau \circ \rho_{1} \in \underline{S}_{c, f}$, we have $\tau \circ \rho_{1} \circ f=\rho_{2} \circ f, \tau \in \operatorname{Symp}_{c}\left(\mathbb{R}^{2}\right)$ and $\tau$ preserves the labelling. Thus $p \circ j$ induces a bijection $\bar{p}: \mathcal{S}_{c}\left(\mathbb{R}^{2}\right) / \underline{S}_{c, f} \rightarrow$ $\operatorname{Diff}_{c}\left(\mathbb{R}^{2}\right) / \sim_{f}$. Then $\bar{p}$ is differentiable. In fact, by Lemma $5.2, \bar{p}$ is induced from the differentiable mapping $\Pi \circ i$. Moreover $\psi: \operatorname{Diff}_{c}\left(\mathbb{R}^{2}\right) / \sim_{f} \rightarrow \mathcal{S}_{c}\left(\mathbb{R}^{2}\right) / \underline{S}_{c, f}$ 
defined by $\psi([\rho]):=\left[\rho^{*} \omega_{0}\right]$ is differentiable and $\psi$ is the inverse of $\bar{p}$. Therefore $\bar{p}$ is a diffeomorphism. For other diffeomorphisms are obtained similarly.

Let us denote by $\mathcal{S}^{+}\left(\mathbb{R}^{2}, y_{0}\right)$ the space of germs of positive symplectic forms on $\left(\mathbb{R}^{2}, y_{0}\right)$. Then similarly to Lemma 5.3 , we have, on local moduli spaces:

Lemma 5.4 (1) For a map-germ $f_{y_{0}}:\left(S^{1}, S\right) \rightarrow\left(\mathbb{R}^{2}, y_{0}\right)$ of finite codimension,

$$
\mathcal{S}^{+}\left(\mathbb{R}^{2}, y_{0}\right) / S_{f_{y_{0}}} \cong \operatorname{Diff}^{+}\left(\mathbb{R}^{2}, y_{0}\right) f / \operatorname{Symp}\left(\mathbb{R}^{2}, y_{0}\right) \cong \mathcal{M}_{\text {symp }}\left(f_{y_{0}}\right)
$$

(2) For a labelled map-germ $f_{y_{0}}:\left(S^{1}, S\right) \rightarrow\left(\mathbb{R}^{2}, y_{0}\right)$ of finite codimension,

$$
\mathcal{S}^{+}\left(\mathbb{R}^{2}, y_{0}\right) / \underline{S}_{f_{y_{0}}} \cong \operatorname{Diff}^{+}\left(\mathbb{R}^{2}, y_{0}\right) / \sim_{f_{y_{0}}}=: \widetilde{\mathcal{M}}_{\mathrm{symp}}\left(f_{y_{0}}\right) .
$$

Proof of Theorem 1.1: Let $f_{0} \in C^{\infty}\left(S^{1}, \mathbb{R}^{2}\right) / \operatorname{Diff}^{+}\left(S^{1}\right)$ be a generic immersion. Define

$$
\Phi: \mathcal{S}_{c}\left(\mathbb{R}^{2}\right) \rightarrow H^{2}\left(\mathbb{R}^{2}, f_{0}\left(S^{1}\right), \mathbb{R}\right)=H_{2}\left(\mathbb{R}^{2}, f_{0}\left(S^{1}\right), \mathbb{R}\right)^{*}
$$

by setting, for $\omega \in \mathcal{S}_{c}\left(\mathbb{R}^{2}\right)$,

$$
\Phi(\omega)\left(\left[D_{j}\right]\right):=\int_{D_{j}} \omega,
$$

$\left(\left[D_{1}\right], \ldots,\left[D_{r}\right] \in H_{2}\left(\mathbb{R}^{2}, f_{0}\left(S^{1}\right), \mathbb{R}\right)\right)$. Then, via the diffeomorphism

$$
\mathcal{S}_{c}\left(\mathbb{R}^{2}\right) / S_{c, f_{0}} \cong \widetilde{\mathcal{M}}_{\mathrm{symp}}(f),
$$

the mapping $\Phi$ induces the mapping $\varphi$ introduced in Introduction. In fact, if $\omega=\rho^{*} \omega_{0}$, then

$$
\int_{D_{j}} \omega=\int_{D_{j}} \rho^{*} \omega_{0}=\int_{\rho\left(D_{j}\right)} \omega_{0} .
$$

Clearly the image of $\Phi$ is contained in the positive cone $H^{2}\left(\mathbb{R}^{2}, f_{0}\left(S^{1}\right), \mathbb{R}\right)_{>0}$. Moreover, we show that

(a): if $\alpha$ is in the image of $\Phi$, and $c>0$, then $c \alpha$ is also in the image of $\Phi$, and (b): if $\alpha$ is in the image of $\Phi$, and $\beta \in H^{2}\left(\mathbb{R}^{2}, f_{0}\left(S^{1}\right), \mathbb{R}\right)_{>0}$, then $\alpha+\beta$ belongs to the image of $\Phi$.

In fact, if $\Phi(\omega)=\alpha$, then $\Phi(c \omega)=c \alpha$. So we have (a).

To show (b), let $\alpha=\Phi(\omega) \in H^{2}\left(\mathbb{R}^{2}, f_{0}\left(S^{1}\right), \mathbb{R}\right)_{>0}$. Let $D$ be one of bounded domains surrounded by $f_{0}\left(S^{1}\right)$. Let $\left(x_{0}, y_{0}\right)$ be an interior point of $D$. Let $\varepsilon>0$ satisfy $D_{\varepsilon}\left(x_{0}, y_{0}\right) \subset D$, where $D_{\varepsilon}\left(x_{0}, y_{0}\right)$ means the $\varepsilon$ closed disc centred at $\left(x_{0}, y_{0}\right)$. Let $\lambda_{\left(x_{0}, y_{0}, \varepsilon\right)}$ be a non-negative $C^{\infty}$ function on $\mathbb{R}^{2}$ with $\operatorname{supp}\left(\lambda_{\left(x_{0}, y_{0}, \varepsilon\right)}\right)=D_{\varepsilon}\left(x_{0}, y_{0}\right)$ and satisfying

$$
\int_{D} \lambda_{\left(x_{0}, y_{0}, \varepsilon\right)} \omega_{0}=1
$$


Set $\lambda_{D}:=\lambda_{\left(x_{0}, y_{0}, \varepsilon\right)}$. Then, for each $\beta \in H^{2}\left(\mathbb{R}^{2}, f_{0}\left(S^{1}\right), \mathbb{R}\right)_{>0}$, set

$$
\omega_{\alpha+\beta}:=\omega+\sum_{j=1}^{r} \beta\left(\left[D_{j}\right]\right) \lambda_{D_{j}} \omega .
$$

Then $\omega_{\alpha+\beta} \in \mathcal{S}_{c}\left(\mathbb{R}^{2}\right)$. Moreover we have

$$
\begin{aligned}
\int_{D_{k}} \omega_{\alpha+\beta} & =\int_{D_{k}} \omega+\sum_{j=1}^{r} \beta\left(D_{j}\right) \int_{D_{k}} \lambda_{D_{j}} \omega \\
& =\alpha\left(\left[D_{k}\right]\right)+\beta\left(\left[D_{k}\right]\right)=(\alpha+\beta)\left(\left[D_{k}\right]\right) .
\end{aligned}
$$

Therefore we have $\Phi\left(\omega_{\alpha+\beta}\right)=\alpha+\beta$. Hence we have (b).

By (a)(b), we see that the image of $\Phi$ coincides with $H^{2}\left(\mathbb{R}^{2}, f_{0}\left(S^{1}\right), \mathbb{R}\right)_{>0}$.

If $\rho \in \underline{S}_{c, f_{0}}$, then $\Phi\left(\rho^{*} \omega\right)=\Phi(\omega)$. Thus $\Phi$ induces the surjective mapping $\varphi: \mathcal{S}_{c}\left(\mathbb{R}^{2}\right) / \underline{S}_{c, f_{0}} \rightarrow H^{2}\left(\mathbb{R}^{2}, f_{0}\left(S^{1}\right), \mathbb{R}\right)_{>0}$.

We will show $\Phi$ is differentiable. To see this, we let $h: P \rightarrow \mathcal{S}_{c}\left(\mathbb{R}^{2}\right)$ be a differentiable mapping. Let a $C^{\infty}$ mapping $H: P \times \mathbb{R}^{2} \rightarrow \bigwedge^{2}\left(T^{*} \mathbb{R}^{2}\right)$ define $h$. Then $\Phi \circ h: P \rightarrow H^{2}\left(\mathbb{R}^{2}, f_{0}\left(S^{1}\right), \mathbb{R}\right)_{>0}$ is defined by

$$
(\Phi \circ h)(p)\left(\left[D_{j}\right]\right):=\int_{D_{j}} h(p)^{*} \omega_{0} .
$$

Therefore $\Phi \circ h$ is differentiable. Since $\mathcal{S}_{c}\left(\mathbb{R}^{2}\right)$ is mild, we see $\Phi$ is differentiable. Therefore $\varphi$ is differentiable.

We are going to show the mapping

$$
\varphi: \mathcal{S}_{c}\left(\mathbb{R}^{2}\right) / \underline{S}_{c, f_{0}} \rightarrow H^{2}\left(\mathbb{R}^{2}, f_{0}\left(S^{1}\right), \mathbb{R}\right)_{>0}
$$

is actually a diffeomorphism. We need to show the surjection $\varphi$ is an injection and its inverse is a differentiable mapping.

Suppose $\Phi(\omega)=\Phi\left(\omega^{\prime}\right)$, for $\omega, \omega^{\prime} \in \mathcal{S}_{c}\left(\mathbb{R}^{2}\right)$. Set $\omega=f(x, y) d x \wedge d y$ and $\omega^{\prime}=g(x, y) d x \wedge d y$. Here $f$ and $g$ are positive functions on $\mathbb{R}^{2}$ which agree with 1 outside a compact subset. Then set $f_{t}:=(1-t) f+t g, 0 \leq t \leq 1$, and $\omega_{t}:=f_{t}(x, y) d x \wedge d y$. Then, by Moser's theorem ([25]), we can find a $C^{\infty}$ family $\rho_{t} \in \underline{S}_{c, f_{0}}$ such that $\omega_{t}=\rho_{t}^{*} \omega_{0}$. Therefore $\omega^{\prime}=\omega_{1}=\rho_{1}^{*} \omega_{0}=\left(\rho_{1} \circ \rho_{0}^{-1}\right)^{*} \omega$. Thus $\varphi$ is an injection. Therefore $\varphi$ is a bijection. Let

$$
\psi:=\varphi^{-1}: H^{2}\left(\mathbb{R}^{2}, f_{0}\left(S^{1}\right), \mathbb{R}\right)_{>0} \rightarrow \mathcal{S}_{c}\left(\mathbb{R}^{2}\right) / \underline{S}_{c, f_{0}}
$$

be the inverse of $\varphi$. We set $\Psi(\alpha+\beta)=\omega_{\alpha+\beta}$ as defined above. Then $\Psi$ is a differentiable map and it gives a local differentiable lifting of $\psi$ on an open set $\{\alpha+\beta \mid \beta>0\}$ for any $\alpha \in H^{2}\left(\mathbb{R}^{2}, f_{0}\left(S^{1}\right), \mathbb{R}\right)_{>0}$. Therefore $\psi$ is differentiable. This shows that $\varphi$ is a diffeomorphism.

Proof of Theorem 1.4: Now let $f \in C^{\infty}\left(S^{1}, \mathbb{R}^{2}\right) / \operatorname{Diff}^{+}\left(S^{1}\right)$ be of finite type. We set

$$
M:=\left(\prod_{y_{0} \in f\left(S^{1}\right)} \mathcal{S}^{+}\left(\mathbb{R}^{2}, y_{0}\right) / \underline{S}_{f_{y_{0}}}\right) \times H^{2}\left(\mathbb{R}^{2}, f\left(S^{1}\right), \mathbb{R}\right)_{>0} .
$$


Define the mapping

$$
\widetilde{\Phi}: \mathcal{S}_{c}\left(\mathbb{R}^{2}\right) \rightarrow M,
$$

by

$$
\begin{aligned}
& \widetilde{\Phi}(\omega):=\left(\left([\omega]_{y_{0}}\right)_{y_{0} \in f\left(S^{1}\right)}, \varphi([\omega])\right), \\
& \varphi([\omega])\left(\left[D_{j}\right]\right)=\int_{D_{j}} \omega, \quad(1 \leq j \leq r) .
\end{aligned}
$$

Then we see $\widetilde{\Phi}$ is differentiable. The mapping $\Phi$ defined in Introduction is given, in term of symplectic forms, by

$$
\Phi: \mathcal{S}_{c}\left(\mathbb{R}^{2}\right) / \underline{S}_{c, f} \rightarrow M, \quad \Phi([\omega])=\widetilde{\Phi}(\omega) .
$$

Note that both $\mathcal{S}_{c}\left(\mathbb{R}^{2}\right) / \underline{S}_{c, f}$ and $M$ are mild quotients (see $\S 3$ ).

We are going to show that $\Phi$ is a diffeomorphism. Since $\widetilde{\Phi}$ is differentiable, we see that $\Phi$ is differentiable.

To see $\Phi$ is injective, suppose that $\Phi\left(\left[\omega_{1}\right]\right)=\Phi\left(\left[\omega_{2}\right]\right)$, for $\omega_{1}, \omega_{2} \in \mathcal{S}_{c}\left(\mathbb{R}^{2}\right)$. Then, for any $y_{0} \in f\left(S^{1}\right),\left[\omega_{1}\right]_{y_{0}}=\left[\omega_{2}\right]_{y_{0}} \in \mathcal{S}^{+}\left(\mathbb{R}^{2}, y_{0}\right) / \underline{S}_{f_{y_{0}}}$, and $\int_{D_{j}} \omega_{1}=$ $\int_{D_{j}} \omega_{2}, 1 \leq j \leq r$. We need to take care of unstable points of $f$, which are a finite number of points by the finiteness of $f$. Set

$$
\mathrm{Uns}(f):=\left\{y_{0} \in f\left(S^{1}\right) \mid f_{y_{0}} \text { is unstable }\right\} .
$$

Then there exist germs of label preserving symmetries $\rho_{y_{0}}:\left(\mathbb{R}^{2}, y_{0}\right) \rightarrow\left(\mathbb{R}^{2}, y_{0}\right)$ of $f_{y_{0}}:\left(S^{1}, S\right) \rightarrow\left(\mathbb{R}^{2}, y_{0}\right)$ such that $\rho_{y_{0}}^{*} \omega_{2}=\omega_{1}$ near $y_{0}$. Note that there exist germs $\sigma_{y_{0}} \in \operatorname{Diff}_{0}^{+}\left(S^{1}, f^{-1}\left(y_{0}\right)\right)$ such that $\rho_{y_{0}} \circ f=f \circ \sigma_{y_{0}}$ near $f^{-1}\left(y_{0}\right)$. The local symmetries $\rho_{y_{0}}, y_{0} \in \operatorname{Uns}(f)$, is covered by a global symmetry $\rho$ of $f$, via a $\sigma \in \operatorname{Diff}^{+}\left(S^{1}\right)$ giving $\sigma_{y_{0}}$ near $f^{-1}\left(y_{0}\right)$. Then $\rho^{*} \omega_{2}=\omega_{1}$ on a neighbourhood of $\operatorname{Uns}(f)$. This construction is extended on a neighborhood of $f\left(S^{1}\right)$. We extend $\rho$ to a $\rho \in \underline{S}_{c, f}$. Now

$$
\int_{D_{j}} \rho^{*} \omega_{2}=\int_{\rho\left(D_{j}\right)} \omega_{2}=\int_{D_{j}} \omega_{2}=\int_{D_{j}} \omega_{1} .
$$

Then there exist a diffeomorphism $\rho^{\prime} \in \underline{S}_{c, f}$ such that $\rho^{\prime}$ is identity on a neighborhood of $f\left(S^{1}\right)$ and $\rho^{\prime *}\left(\rho^{*} \omega_{2}\right)=\omega_{1}$. Now $\rho \circ \rho^{\prime} \in \underline{S}_{c, f}$, so we have $\left[\omega_{1}\right]=\left[\omega_{2}\right]$. Therefore we see that $\Phi$ is an injection.

Next we show $\Phi$ is surjective. Take a germ of positive form $\omega_{y_{0}}=f_{y_{0}}(x, y) d x \wedge$ $d y$ at every $y_{0} \in \operatorname{Uns}(f)$. Then, for any given $\varepsilon_{j}>0,1 \leq j \leq r,\left(\omega_{y_{0}}\right)_{y_{0} \in \operatorname{Uns}(f)}$ is extended to $\omega \in \mathcal{S}_{c}\left(\mathbb{R}^{2}\right)$ such that $\int_{D_{j}} \omega<\varepsilon_{j}, 1 \leq j \leq r$. This is established by setting

$$
\omega=\left[\sum_{y_{0} \in \operatorname{Uns}(f)} \mu_{y_{0}}(x, y) f_{y_{0}}(x, y)+\varepsilon(x, y)\right] d x \wedge d y,
$$


using a non-negative $C^{\infty}$ functions $\mu_{y_{0}}(x, y)$ and $\varepsilon$ on $\mathbb{R}^{2}$ for $y_{0} \in \operatorname{Uns}(f)$ satisfying the following conditions: (i) $\mu_{y_{0}}(x, y)$ is equal to 1 in a neighbourhood of $y_{0}$ and $\varepsilon$ is equal to 0 in a smaller neighborhood of the finite set $\operatorname{Uns}(f)$. (ii) $\mu_{y_{0}}(x, y)$ is equal to 0 in a neighbourhood of $y_{0}$ and $\varepsilon$ is sufficiently small on each $D_{j}$, so that $\int_{D_{j}} \omega<\varepsilon_{j}$. (iii) $\varepsilon$ is equal to 1 outside of a compact set.

In Fig. 3, we illustrate a required extension $\omega$ of given form-germs $\omega_{y_{0}}$. Then, for any $\alpha \in H^{2}\left(\mathbb{R}^{2}, f\left(S^{1}\right), \mathbb{R}\right)_{>0}$ with $\alpha\left(D_{j}\right)>\varepsilon_{j}$, set

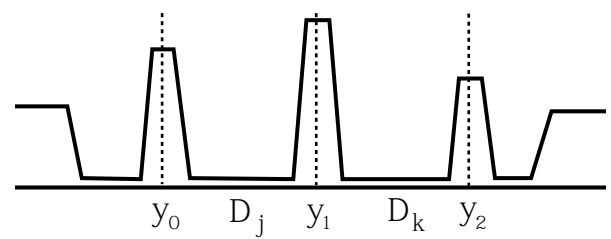

Figure 3: Extending form-germs to the plane.

$$
\omega_{\left(\left(\omega_{y_{0}}\right), \alpha\right)}=\omega+\left(\alpha\left(D_{j}\right)-\int_{D_{j}} \omega\right) \lambda_{D_{j}} d x \wedge d y .
$$

Here $\lambda_{D_{j}}$ is a bump function as introduced in the proof of Theorem 1.1. Then $\omega_{\left(\left(\omega_{y_{0}}\right), \alpha\right)} \in \mathcal{S}_{c}\left(\mathbb{R}^{2}\right)$ and

$$
\widetilde{\Phi}\left(\omega_{\left(\omega_{y_{0}}\right), \alpha}\right)=\left(\left(\omega_{y_{0}}\right), \alpha\right)
$$

Since $\varepsilon_{j}>0$ is arbitrary, we see $\widetilde{\Phi}$ is surjective. Thus we see $\Phi$ is bijective.

Let $\Psi: M \rightarrow \mathcal{S}_{c}\left(\mathbb{R}^{2}\right) / \underline{S}_{c, f}$ be the inverse of $\Phi$. We are going to show $\Psi$ is differentiable. Let

$$
h: P \rightarrow\left(\prod_{y_{0} \in \operatorname{Uns}(f)} \mathcal{S}^{+}\left(\mathbb{R}^{2}\right)\right) \times H^{2}\left(\mathbb{R}^{2}, f\left(S^{1}\right), \mathbb{R}\right)_{>0}
$$

be a differentiable mapping from a finite dimensional manifold $P$. We ask whether $\Psi \circ h: P \rightarrow \mathcal{S}_{c}\left(\mathbb{R}^{2}\right) / \underline{S}_{c, f}$ is differentiable. Let a $C^{\infty}$ mapping

$$
H: P \times\left(\coprod_{y_{0} \in \mathrm{Uns}(f)} \mathbb{R}^{2}\right) \coprod\{\mathrm{pt}\} \rightarrow\left(\coprod_{y_{0} \in \mathrm{Uns}(f)} \mathbb{R}\right) \coprod H^{2}\left(\mathbb{R}^{2}, f\left(S^{1}\right), \mathbb{R}\right)_{>0}
$$

defines $h$, where $\coprod_{y_{0} \in \mathrm{Uns}(f)} \mathbb{R}^{2}$ means the disjoint union of $r^{\prime \prime}$-copies of $\mathbb{R}^{2}, r^{\prime \prime}$ being the number of $\operatorname{Uns}(f)$. We are identifying $\mathbb{R}$ with $\bigwedge^{2}\left(\mathbb{R}^{2}\right)^{*}$. Set

$$
H\left(\lambda, y_{1}, \ldots, y_{r^{\prime \prime}}, \mathrm{pt}\right)=\left(\left(\omega_{y_{0}, \lambda}=f_{y_{0}}(x, y, \lambda) d x \wedge d y\right)_{y_{0} \in \operatorname{Uns}(f)}, \quad \alpha_{\lambda}\right) .
$$

For each compact subset $\Lambda$ of $P$, we construct a $C^{\infty}$ family $\omega_{\lambda} \in \mathcal{S}_{c}\left(\mathbb{R}^{2}\right)$ by

$$
\omega_{\left(\left(\omega_{y_{0}, \lambda}\right), \alpha_{\lambda}\right)}:=\omega+\sum_{j=1}^{r}\left(\alpha\left(D_{j}\right)-\int_{D_{j}} \omega\right) \lambda_{D_{j}} d x \wedge d y
$$


where

$$
\omega:=\left[\sum_{y_{0} \in \operatorname{Uns}(f)} \mu_{y_{0}}(x, y) f_{y_{0}}(x, y, \lambda)+\varepsilon(x, y)\right] d x \wedge d y
$$

is defined by functions $\mu_{y_{0}}$ and $\varepsilon(x, y)$ which are independent of $\lambda \in \Lambda$. Then

$$
\widetilde{\Psi \circ h}\left(\left(\omega_{y_{0}}, \lambda\right), \alpha_{\lambda}\right):=\omega_{\left(\left(\omega_{y_{0}, \lambda}\right), \alpha_{\lambda}\right)}
$$

gives a local differentiable lifting of $\Psi \circ h$. Therefore $\Psi \circ h$ is also differentiable. Since $M$ is a mild quotient, we see $\Psi$ is differentiable. Thus we have shown that $\Phi$ is a diffeomorphism as required.

Proof of Theorems 1.8 and 1.9: The proofs are established in the parallel way to that of Theorem 1.4. Therefore they are left to the reader.

\section{References}

[1] V.I. Arnold, Topological invariants of plane curves and caustics, University Lecture Series, 5 Amer. Math. Soc., Providence, RI, (1994).

[2] J.-L. Brylinski, Loop Spaces, Characteristic Classes and Geometric Quantization, Prog. in Math., 107, Birkhäuser (1993).

[3] J.W. Bruce, T. Gaffney, Simple singularities of mappings $\mathbb{C}, 0 \rightarrow \mathbb{C}^{2}, 0$, J. London Math. Soc., 26 (1982), 465-474.

[4] W. Domitrz, S. Janeczko, M. Zhitomirskii, Relative Pincaré lemma, contractibility, quasi-homogeneity and vector fields tangent to a singular variety, Illinois J. Math. 48-3 (2004), 803-835.

[5] W. Domitrz, J.H. Rieger, Volume-preserving diffeomorphisms on varieties and $\mathcal{A}_{\Omega^{-}}$ equivalence of maps, Preprint (2006).

[6] S. Ebey, The classification of singular points of algebraic curves, Trans. Amer. Math. Soc., 118 (1965), 454-471.

[7] J. Eells, Jr., On the geometry of functions spaces, International symposium on algebraic topology, Universidad Nacional Autónoma de México and UNESCO, Mexico City, (1958), pp. 303-308.

[8] M.D. Garay, An isochore versal deformation theorem, Topology, 43 (2004), 1081-1088.

[9] M. Golubitsky, V. Guillemin, Stable Mappings and their Singularities, Graduate Text in Math., 14 Springer-Verlag, New York (1973).

[10] R. Greene, K. Shiohama, Diffeomorphisms and volume preserving embedding of noncompact manifolds, Trans. Amer. Math. Soc., 225 (1979), 403-414.

[11] A.B. Givental, Singular Lagrangian varieties and their Lagrangian mappings, in Itogi Nauki Tekh., Ser. Sovrem. Probl. Mat., (Contemporary Problems of Mathematics) 33, VITINI, (1988), pp. 55-112.

[12] A. Hefez, M.E. Hernandes, Classification of algebroid plane curves with semigroup $\langle 6,9,19\rangle^{\#}$, Comm. in Algebra, 31-8 (2003), 3847-3861.

[13] N.J. Hitchin, The moduli space of special Lagrangian submanifolds, Ann. Scuola Norm. Sup. Pisa, 25 no. 3-4 (1997), 503-515 (1998).

[14] N.J. Hitchin, The moduli space of complex Lagrangian submanifolds, Asian J. Math., 3-1 (1999), 77-91. 
[15] G. Ishikawa, Families of functions dominated by distributions of $\mathcal{C}$-classes of mappings, Ann. Inst. Fourier, 33-2 (1983), 199-217.

[16] G. Ishikawa, Symplectic and Lagrange stabilities of open Whitney umbrellas, Invent. math., 126-2 (1996), 215-234.

[17] G. Ishikawa, S. Janeczko, Symplectic bifurcations of plane curves and isotropic liftings, Quart. J. Math., 54 (2003), 1-30.

[18] G. Ishikawa, S. Janeczko, Symplectic singularities of isotropic mappings, in Geometric Singularity Theory, Banach Center Publications 65, eds.: Heisuke Hironaka, Stanislaw Janeczko, Stanislaw Lojasiewicz. (2004), pp. 85-106.

[19] G. Ishikawa, S. Janeczko, The complex symplectic moduli spaces of parametric plane curve singularities, Preprint (2006).

[20] S. Izumi, Private communication.

[21] S.K. Lando, Normal forms of the degrees of a volume form, Funct. Anal. Appl. 19-2 (1984), 146-148.

[22] O.A. Laudal, G. Pfister, Local moduli and singularities, Lecture Notes in Math., 1310 Springer-Verlag, Berlin (1988)

[23] I. Luengo, G. Pfister, Normal forms and moduli spaces of curve singularities with semigroup $\langle 2 p, 2 q, 2 p q+d\rangle$, Comp. Math., 76 (1990), 247-264.

[24] J.N. Mather, Stability of $C^{\infty}$ mappings III: Finitely determined map-germs, Publ. Math. I.H.E.S., 35 (1968), 127-156.

[25] J. Moser, On the volume elements on a manifolds, Trans. Amer. Math. Soc., 120-2 (1965), 286-294.

[26] H. Omori, Infinite-dimensional Lie groups, Translations of Mathematical Monographs, 158 Amer. Math. Soc., (1997)

[27] B. Teissier, Appendix in [32].

[28] J.-Cl. Tougeron, Idéaux de fonctions différentiables, Ergebnisse der Mathematik, 71 Springer-Verlag, (1972).

[29] A.N. Varchenko, Local classification of volume forms in the presence of a hypersurface, Funct. Anal. Appl. 19-4 (1984), 269-276.

[30] J.M. Wahl, Equisingular deformations of plane algebroid curves, Trans. Amer. Math. Soc., 193 (1974), 143-170.

[31] C.T.C. Wall, Finite determinacy of smooth map-germs, Bull. London Math. Soc., 13 (1981), 481-539.

[32] O. Zariski, Le problème des modules pour les branches planes, Cours donné au Centre de mathématiques de l'École Polytechnique, 1973, (ed. F. Kmety, M. Merle, with an appendix of B. Tessier), Hermann, Paris (1987).

[33] M. Zhitomirskii, Germs of integral curves in contact 3-space, plane and space curves, Isaac Newton Inst. preprint NI00043-SGT, December 2000.

[34] M. Zhitomirskii, Relative Darboux theorem for singular manifolds and local contact algebra, Canad. J. Math., 57-6 (2005), 1314-1340.

\section{Goo ISHIKAWA}

Department of Mathematics, Hokkaido University, Sapporo 060-0810, Japan.

E-mail : ishikawa@math.sci.hokudai.ac.jp

Web: http://www.math.sci.hokudai.ac.jp/ ishikawa 